\title{
A Novel Coupled State/Input/Parameter Identification Method for Linear Structural Systems
}

\author{
Zhimin Wan $\left(\mathbb{D},{ }^{1,2}\right.$ Ting Wang $\mathbb{D},{ }^{3} \mathrm{Lin} \mathrm{Li},{ }^{2}$ and Zhichao $\mathrm{Xu}{ }^{2}$ \\ ${ }^{1}$ School of Vehicle and Transportation Engineering, Nantong Vocational University, Qingnian Middle Road No. 89, Nantong, China \\ ${ }^{2}$ State Key Laboratory of Digital Manufacturing Equipment and Technology, Huazhong University of Science and Technology, \\ Wuhan 430074, China \\ ${ }^{3}$ School of Mechanical Engineering, Nantong Vocational University, Qingnian Middle Road No. 89, Nantong, China
}

Correspondence should be addressed to Ting Wang; lwxting@hust.edu.cn

Received 18 August 2017; Revised 30 November 2017; Accepted 23 January 2018; Published 20 February 2018

Academic Editor: Tony Murmu

Copyright (c) 2018 Zhimin Wan et al. This is an open access article distributed under the Creative Commons Attribution License, which permits unrestricted use, distribution, and reproduction in any medium, provided the original work is properly cited.

\begin{abstract}
In many engineering applications, unknown states, inputs, and parameters exist in the structures. However, most methods require one or two of these variables to be known in order to identify the other(s). Recently, the authors have proposed a method called EGDF for coupled state/input/parameter identification for nonlinear system in state space. However, the EGDF method based solely on acceleration measurements is found to be unstable, which can cause the drift of the identified inputs and displacements. Although some regularization methods can be adopted for solving the problem, they are not suitable for joint input-state identification in real time. In this paper, a strategy of data fusion of displacement and acceleration measurements is used to avoid the low-frequency drift in the identified inputs and structural displacements for linear structural systems. Two numerical examples about a plane truss and a single-stage isolation system are conducted to verify the effectiveness of the proposed modified EGDF algorithm.
\end{abstract}

\section{Introduction}

The state of a system is vital to structural health monitoring in mechanical engineering and civil engineering. The topic of identifying the state has been studied intensively for many decades. Various state estimators [1-7] for structural systems behaving both linearly and nonlinearly have been proposed, including the well-known Kalman filter (KF) [8] for linear structural systems.

However, only adopting KF to identify the structural state is often insufficient due to the uncertain structural parameters. The extended Kalman filter (EKF) [9], used for slightly nonlinear system, was proposed by linearizing the dynamic model. EKF has been one of the most widely used tools for joint input/parameter identification in structural dynamics, and it has been adopted in many applications, such as damage identification [10,11], parameter identification $[12,13]$, and model updating [14].

In all of the above EKF applications, the input forces are assumed to be known. Unfortunately, the measurement of the input forces on a structure is not straightforward, because the introduction of dedicated force cells requires alteration to the structure to locate the sensor in the force path, which is unwanted and unpractical. The indirect measurement of the input forces, like calibrated strain gauges, requires a good knowledge of the structural parameters, which might be unknown as well. On the other hand, input forces together with the system states can be identified through a variety of $\mathrm{KF}$ approaches and acceleration measurements, which have gained increasing attention in recent years. Gillijns and De Moor [15] proposed a recursive optimal filter of joint state/input estimation for linear systems with direct transmission, which was originally presented for optimal control applications. The estimated input and state are optimal in a minimum-variance unbiased sense. Lourens et al. [16] extended this filter proposed by Gillijns and De Moor (GDF) of joint input/response identification for structural systems based on reduced-order model and acceleration data from a limited number of sensors. Maes et al. [17, 18] presented an extension of the GDF algorithm for joint input/state 
estimation in structural dynamics, accounting for the correlation between process noise and measurement noise. In [19], an augmented Kalman filter for joint state/force identification in structural dynamics was presented, in which the unknown forces and states were reformed into augmented states. And the KF was employed for identifying the novel augmented states. Naets et al. [20] adopted a similar algorithm for identifying the states and forces of multibody model. The difference is that EKF is employed for the joint state/input identification. In [21], the Popov-Belevitch-Hautus (PBH) criterion was used to evaluate the observation of the system for the augmented KF algorithm. It was shown that only using acceleration measurements inherently would bring unreliable results. To avoid this deficiency, the addition of dummy measurements on a position level was proposed. In [22, 23], a DKF (Dual KF) approach of joint state/input identification was proposed for linear state-space models. These methods in this paragraph associated with joint state/input identification are based on an accurate structural model in which there are no uncertain parameters. However, it is still impossible to obtain a completely accurate model due to the structural complexity and the manufacturing or measuring errors.

From the above literatures, it is known that the identification of forces and parameters cannot be separated for general structures. For improvement, several approaches have been developed for joint input/parameter identification. Kolmanovsky et al. [24] developed the coupled identification of an input and set-membership parameter while this algorithm requires fully known states, which is rarely the case in engineering applications. Yang et al. [25] proposed an EKF with unknown input forces, referred to as EKFUI, for the coupled state/input/parameter identification for structural damage detection. Recently, Lei et al. [26] applied the least-squares estimation algorithm to deduce the EKF-UI algorithm again and pointed out that the analytical recursive solutions by the original EKF-UI were acquired by relatively complex mathematical derivations. Lei et al. [27] also applied the above simplified EKF-UI algorithm for nonlinear structural parameter identification. However, in the simplified EKF-UI algorithm, the prior probability density functions (PDFs) of states at $t+1$ time are taken as the posterior PDFs of states at $t+1$ time, which may cause the confusion of concepts in Bayesian framework. Another method called augmented discrete extended Kalman filter (A-DEKF) for the coupled state/input/parameter identification was proposed by Naets et al. [28]. A-DEKF is similar to the augmented KF algorithm [19] where a high dimensional augmented state vector was formed by the states, unknown forces, and uncertain parameters. Moreover, a suitable assumption on the statistics of the force is needed for the A-DEKF method. In [29], the same methodology is also applied to an offshore wind turbine in order to simultaneously estimate the hydrodynamic loading, states, and a stiffness-related system parameter. Recently, a DKF-UKF framework has been developed for the coupled identification topic in [30, 31]. A DKF is used to identify the input, and an UKF (Unscented $\mathrm{KF}$ ) is adopted to identify both the states and the unknown parameters (the so-called augment states). However, the stability, observability, and convergence analysis of DKF-UKF are not mentioned. Also a prior assumption on the covariance of the input is needed in DKF, which strongly influences the quality of the estimates of the Bayesian filters. The authors also proposed a method based on the GDF algorithm for coupled state/input/parameter identification for nonlinear systems [32]. The EGDF (extended GDF) algorithm is developed for the weak nonlinear systems by the linearization idea of EKF. The uncertain parameters and states are considered as the augmented states to be identified. Thus, the novel state transmission and measurement equations become nonlinear, which are linearized by the first-order Taylor expansions. The proposed EGDF has the same structure of the standard GDF algorithm, including three steps: input identification, measurement update, and time update. The main difference between them is the sensitivity matrices of the two nonlinear state-space equations. However, it has been demonstrated that the conventional GDF approach based on limited number of acceleration measurements is inherently unstable which leads to the so-called spurious low-frequency drift in the estimates of the force and the structural displacement [22]. Although some regularization methods or postsignal processing schemes can be adopted to deal with the drift in the identified results [33-36], they prohibit the real-time identification of coupled state/input/parameter.

In this paper, a strategy of data fusion of partially measured displacement and acceleration responses is adopted to avoid the so-called drifts in the identified displacement and force in the conventional GDF approach, since displacement and acceleration measurements contain low and high frequencies vibration characteristics, respectively.

This paper proceeds as follows. In Section 2, the standard GDF algorithm for joint state/input identification is presented. In Section 3, the nonlinear identification model of the coupled state/input/parameter is firstly built, and then the EGDF method is demonstrated, including data fusion of partially measured acceleration and displacement responses. Section 4 conducts two numerical examples to demonstrate the effectiveness of the modified EGDF method. Finally, the conclusion is drawn.

\section{Joint State/Input Identification}

2.1. Discrete-Time State-Space Model of Structural Dynamics. The general equation of motion of a damped structure with $n$ DOFs can be expressed as

$$
\mathbf{M} \ddot{\mathbf{p}}(t)+\mathbf{C} \dot{\mathbf{p}}(t)+\mathbf{K} \mathbf{p}(t)=\mathbf{B}_{u} \mathbf{u}(t),
$$

where $\mathbf{M}, \mathbf{C}$, and $\mathbf{K}$ are the mass, damping, and stiffness matrices of the structure, respectively; $\ddot{\mathbf{p}}(t), \dot{\mathbf{p}}(t)$, and $\mathbf{p}(t)$ are, respectively, the nodal acceleration, velocity, and displacement vectors of the structure; $\mathbf{u}(t)$ is the force vector and $\mathbf{B}_{u}$ is the influence matrix associated with the input $\mathbf{u}(t)$.

The second-order equation of motion (1) can be transformed into a first-order continuous-time equation in state space as

$$
\dot{\mathbf{x}}(t)=\mathbf{A}_{c} \mathbf{x}(t)+\mathbf{B}_{c} \mathbf{u}(t),
$$


where $\mathbf{x}(t)$ is the state vector, and the system matrices together with $\mathbf{x}(t)$ are, respectively, defined as

$$
\begin{aligned}
\mathbf{A}_{c} & =\left[\begin{array}{cc}
{[\mathbf{0}]} & \mathbf{I} \\
-\mathbf{M}^{-1} \mathbf{K} & -\mathbf{M}^{-1} \mathbf{C}
\end{array}\right], \\
\mathbf{B}_{c} & =\left[\begin{array}{c}
{[\mathbf{0}]} \\
\mathbf{M}^{-1} \mathbf{B}_{u}
\end{array}\right], \\
\mathbf{x}(t) & =\left[\begin{array}{l}
\mathbf{p}(t) \\
\dot{\mathbf{p}}(t)
\end{array}\right] .
\end{aligned}
$$

Assuming that only acceleration responses are measured, the measurement equation can be changed into the following state-space form:

$$
\mathbf{y}(t)=\mathbf{H x}(t)+\mathbf{D u}(t),
$$

with the output influence matrix $\mathbf{H}$ and direct transmission matrix $\mathbf{D}$ defined as

$$
\begin{aligned}
& \mathbf{H}=\left[-\mathbf{H}_{a} \mathbf{M}^{-1} \mathbf{K}-\mathbf{H}_{a} \mathbf{M}^{-1} \mathbf{C}\right], \\
& \mathbf{D}=\left[\mathbf{H}_{a} \mathbf{M}^{-1} \mathbf{B}_{u}\right],
\end{aligned}
$$

where $\mathbf{H}_{a}$ represents the selection matrix of acceleration measurements.

The time step size is denoted as the symbol $\Delta t$; thus the continuous-time state-space model of (2) and (4) can be transformed into the discrete-time form using a sampling rate of $1 / \Delta t$ as

$$
\begin{aligned}
\mathbf{x}_{k+1} & =\mathbf{A} \mathbf{x}_{k}+\mathbf{B} \mathbf{u}_{k}, \\
\mathbf{y}_{k} & =\mathbf{H} \mathbf{x}_{k}+\mathbf{D} \mathbf{u}_{k},
\end{aligned}
$$

where

$$
\begin{aligned}
& \mathbf{x}_{k}=\mathbf{x}(k \Delta t), \\
& \mathbf{y}_{k}=\mathbf{y}(k \Delta t), \\
& \mathbf{A}=e^{\mathbf{A}_{c} \Delta t}, \\
& \mathbf{B}=\left(e^{\mathbf{A}_{c} \Delta t}-\mathbf{I}\right)\left(\mathbf{A}_{c}\right)^{-1} \mathbf{B}_{c} .
\end{aligned}
$$

2.2. The GDF Algorithm for Joint State-Input Identification. Adding the system and measurement noise to the linear structural system, the system equations can be rewritten as

$$
\begin{aligned}
\mathbf{x}_{k+1} & =\mathbf{A} \mathbf{x}_{k}+\mathbf{B} \mathbf{u}_{k}+\mathbf{w}_{k}=f_{k}\left(\mathbf{x}_{k}, \mathbf{u}_{k}\right)+\mathbf{w}_{k}, \\
\mathbf{y}_{k} & =\mathbf{H} \mathbf{x}_{k}+\mathbf{D} \mathbf{u}_{k}+\mathbf{v}_{k}=h_{k}\left(\mathbf{x}_{k}, \mathbf{u}_{k}\right)+\mathbf{v}_{k} .
\end{aligned}
$$

The system noise vector $\mathbf{w}_{k}$ and measurement noise vector $\mathbf{v}_{k}$ are assumed to be mutually uncorrelated, zero-mean, white random signal with known covariance matrices, $\mathbf{G}_{k}$ and $\mathbf{R}_{k}$, respectively. Results are easily generalized to the case where $\mathbf{w}_{k}$ and $\mathbf{v}_{k}$ are correlated [37]. Results can also be easily generalized to systems with both known and unknown input. It is obviously seen that (8) represent the linear system of structural dynamics with direct feedthrough. In the field of system control, Gillijns and De Moor proposed an optimal recursive filter of the direct feedthrough case for joint input/state identification [15]. In this filter, a state estimate $\mathbf{x}_{k \mid l}$ is defined as an estimate of $\mathbf{x}_{k}$ given $\left\{\mathbf{y}_{n}\right\}_{n=0}^{l}$ and its error covariance matrix $\mathbf{P}_{k \mid l}$ expressed as $E\left[\left(\mathbf{x}_{k}-\mathbf{x}_{k \mid l}\right)\left(\mathbf{x}_{k}-\mathbf{x}_{k \mid l}\right)^{T}\right]$. An initial unbiased state estimate $\mathbf{x}_{0 \mid-1}$ and its covariance matrix $\mathbf{P}_{0 \mid-1}^{x}$ are assumed known.

The GDF algorithm computes the unknown force and state in a recursive procedure including three steps: the step of the input identification, the measurement update, and the time update. The GDF algorithm is listed in Algorithm 1.

In [16], the GDF is adopted in linear structural dynamics for joint input/state identification successfully. However, it is not suitable for nonlinear dynamic systems.

\section{Coupled State/Input/Parameter Identification for Linear Systems}

In this section, uncertain structure with unknown structural parameters is considered to identify both state and input. Therefore, a novel more difficult problem of coupled state/ input/parameter identification is presented for more common engineering applications.

3.1. Nonlinear Identification Model of the Coupled State/Input/ Parameter Identification. In dynamics Eq. (1), it is assumed that only $\mathbf{K}$ and $\mathbf{C}$ contain the uncertain parameters $\boldsymbol{\alpha}$ to be identified; then the original state vector $\mathbf{x}$ can be extended to be the augmented state vector as

$$
\mathbf{z}(t)=\left[\begin{array}{c}
\mathbf{x}(t) \\
\boldsymbol{\alpha}
\end{array}\right]
$$

where $\boldsymbol{\alpha}=\left[\begin{array}{llll}\alpha_{1} & \alpha_{2} & \cdots & \alpha_{\alpha}\end{array}\right]^{T}$ is the parameter vector. Therefore, the state transmission and measurement equations can be changed into the following form as

$$
\begin{aligned}
\dot{\mathbf{z}}(t) & =\left[\begin{array}{c}
\dot{\mathbf{x}}(t) \\
\dot{\boldsymbol{\alpha}}
\end{array}\right]=\left[\begin{array}{c}
\dot{\mathbf{p}}(t) \\
\ddot{\mathbf{p}}(t) \\
{[\mathbf{0}]}
\end{array}\right] \\
& =\left[\begin{array}{c}
\dot{\mathbf{p}}(t) \\
-\mathbf{M}^{-1} \mathbf{K} \mathbf{p}(t)-\mathbf{M}^{-1} \mathbf{C} \dot{\mathbf{p}}(t)+\mathbf{M}^{-1} \mathbf{B}_{u} \mathbf{u}(t) \\
{[\mathbf{0}]}
\end{array}\right] \\
& \equiv f^{c}(\mathbf{z}(t), \mathbf{u}(t)), \\
\mathbf{y}(t) & =\mathbf{H x}(t)+\mathbf{D u}(t) \equiv h(\mathbf{z}(t))+\mathbf{D u}(t) .
\end{aligned}
$$

The unknown parameters are assumed to be constant; that is, $\dot{\boldsymbol{\alpha}}=[\mathbf{0}]$. From these two equations, it is found that the identification problem now becomes nonlinear even if the original dynamics system is linear due to the nonlinear coupling of the state $\mathbf{x}$ with $\boldsymbol{\alpha}$. 
The above continuous-time state-space equations can be transformed into the discrete-time form as

$$
\begin{aligned}
\mathbf{z}_{k+1} & =f_{k}\left(\mathbf{z}_{k}, \mathbf{u}_{k}\right)+\mathbf{w}_{k} \quad k=1,2, \ldots, T, \\
\mathbf{y}_{k} & =h_{k}\left(\mathbf{z}_{k}\right)+\mathbf{D}_{k} \mathbf{u}_{k}+\mathbf{v}_{k} \quad k=1,2, \ldots, T .
\end{aligned}
$$

Unlike (8), the functions $f$ and $h$ are nonlinear. The standard GDF method cannot be used to deal with this case of nonlinear identification. In the field of system control, the standard KF algorithm was presented for identifying the state of linear dynamic systems. The EKF algorithm was then developed for identifying the state of nonlinear dynamic systems by continually updating a linearization around the previous state estimate, starting with an initial guess, which is a nonlinear variation on the standard KF based on the first-order Taylor approximation. In the following section, the linearization idea of the EKF method is used to extend the standard GDF algorithm for nonlinear dynamic systems.

3.2. The Extended GDF Algorithm. For this coupled state/ input/parameter identification, the extended state $\mathbf{z}_{k}$ (including structural state and uncertain parameters) and identified forces $\mathbf{u}_{k}$ are both unknown. Therefore, we have the firstorder Taylor series expansions for the two nonlinear system matrices

$$
\begin{aligned}
f_{k}\left(\mathbf{z}_{k}, \mathbf{u}_{k}\right)= & f_{k}\left(E\left(\mathbf{z}_{k}\right), E\left(\mathbf{u}_{k}\right)\right)+\nabla_{\mathbf{z}} f_{k} \\
& \cdot\left(\mathbf{z}_{k}-E\left(\mathbf{z}_{k}\right)\right)+\nabla_{\mathbf{u}} f_{k} \cdot\left(\mathbf{u}_{k}-E\left(\mathbf{u}_{k}\right)\right) \\
& + \text { HOT, } \\
h_{k}\left(\mathbf{z}_{k}\right)= & h_{k}\left(E\left(\mathbf{z}_{k}\right)\right)+\nabla_{\mathbf{z}} h_{k} \cdot\left(\mathbf{z}_{k}-E\left(\mathbf{z}_{k}\right)\right) \\
& + \text { HOT, }
\end{aligned}
$$

where $\nabla_{\mathbf{z}} f_{k}, \nabla_{\mathbf{u}} f_{k}$, and $\nabla_{\mathbf{z}} h_{k}$ are the sensitivity matrices, which are derived in the following text. And HOT represents the high-order terms.

With the Taylor expansions, (13) can be approximated in the following form as

$$
\begin{aligned}
\mathbf{z}_{k}= & f_{k-1}\left(\mathbf{z}_{k-1}, \mathbf{u}_{k-1}\right)+\mathbf{w}_{k-1} \\
= & f_{k-1}\left(E\left(\mathbf{z}_{k-1}\right), E\left(\mathbf{u}_{k-1}\right)\right)+\nabla_{\mathbf{z}} f_{k-1} \\
& \cdot\left(\mathbf{z}_{k-1}-E\left(\mathbf{z}_{k-1}\right)\right)+\nabla_{\mathbf{u}} f_{k-1} \cdot\left(\mathbf{u}_{k-1}-E\left(\mathbf{u}_{k-1}\right)\right) \\
& +\mathbf{w}_{k-1}+\mathrm{HOT} \\
\approx & f_{k-1}\left(E\left(\mathbf{z}_{k-1}\right), E\left(\mathbf{u}_{k-1}\right)\right)+\nabla_{\mathbf{z}} f_{k-1} \\
& \cdot\left(\mathbf{z}_{k-1}-E\left(\mathbf{z}_{k-1}\right)\right)+\nabla_{\mathbf{u}} f_{k-1} \cdot\left(\mathbf{u}_{k-1}-E\left(\mathbf{u}_{k-1}\right)\right) \\
& +\mathbf{w}_{k-1}=\mathbf{z}_{k}^{\operatorname{lin}}, \\
\mathbf{y}_{k}= & h_{k}\left(E\left(\mathbf{z}_{k}\right)\right)+\nabla_{\mathbf{z}} h_{k} \cdot\left(\mathbf{z}_{k}-E\left(\mathbf{z}_{k}\right)\right)+\mathrm{HOT} \\
& +\mathbf{D}_{k} \mathbf{u}_{k}+\mathbf{v}_{k} \\
\approx & \nabla_{\mathbf{z}} h_{k} \cdot \mathbf{z}_{k}+\mathbf{D}_{k} \mathbf{u}_{k}+h_{k}\left(E\left(\mathbf{z}_{k}\right)\right)-\nabla_{\mathbf{z}} h_{k} \cdot E\left(\mathbf{z}_{k}\right) \\
& +\mathbf{v}_{k}=\mathbf{y}_{k}^{\operatorname{lin}} .
\end{aligned}
$$

For the time update step, the goal is to find linear approximations of $\mathbf{z}_{k \mid k-1}$ and $\mathbf{P}_{k \mid k-1}^{z}$. We expand $f_{k-1}\left(\mathbf{z}_{k-1}, \widehat{\mathbf{u}}_{k-1}\right)$ in the neighborhood of $\mathbf{z}_{k-1 \mid k-1}$. With the linear approximation, we get

$$
\begin{aligned}
& \mathbf{z}_{k \mid k-1} \equiv E\left(\mathbf{z}_{k} \mid \mathbf{Y}_{k-1}\right) \approx E\left(f_{k-1}\left(E\left(\mathbf{z}_{k-1}\right), E\left(\mathbf{u}_{k-1}\right)\right)\right. \\
& \quad+\nabla_{\mathbf{z}} f_{k-1} \cdot\left(\mathbf{z}_{k-1}-E\left(\mathbf{z}_{k-1}\right)\right)+\nabla_{\mathbf{u}} f_{k-1} \\
& \left.\cdot\left(\mathbf{u}_{k-1}-E\left(\mathbf{u}_{k-1}\right)\right)+\mathbf{w}_{k-1} \mid \mathbf{Y}_{k-1}\right) \\
& =f_{k-1}\left(\mathbf{z}_{k-1 \mid k-1}, \widehat{\mathbf{u}}_{k-1}\right)=\mathbf{z}_{k \mid k-1}^{\operatorname{lin}} .
\end{aligned}
$$
given by

$$
\begin{aligned}
\widetilde{\mathbf{z}}_{k \mid k-1} \equiv & \mathbf{z}_{k}-\mathbf{z}_{k \mid k-1} \approx \mathbf{z}_{k}^{\operatorname{lin}}-\mathbf{z}_{k \mid k-1}^{\operatorname{lin}} \\
= & f_{k-1}\left(E\left(\mathbf{z}_{k-1}\right), E\left(\mathbf{u}_{k-1}\right)\right)+\nabla_{\mathbf{z}} f_{k-1} \\
& \cdot\left(\mathbf{z}_{k-1}-E\left(\mathbf{z}_{k-1}\right)\right)+\nabla_{\mathbf{u}} f_{k-1} \\
& \cdot\left(\mathbf{u}_{k-1}-E\left(\mathbf{u}_{k-1}\right)\right)+\mathbf{w}_{k-1} \\
& -f_{k-1}\left(E\left(\mathbf{z}_{k-1 \mid k-1}\right), E\left(\widehat{\mathbf{u}}_{k-1}\right)\right)-\nabla_{\mathbf{z}} f_{k-1} \\
& \cdot\left(\mathbf{z}_{k-1 \mid k-1}-E\left(\mathbf{z}_{k-1 \mid k-1}\right)\right)-\nabla_{\mathbf{u}} f_{k-1} \\
& \cdot\left(\widehat{\mathbf{u}}_{k-1}-E\left(\widehat{\mathbf{u}}_{k-1}\right)\right) .
\end{aligned}
$$

Since $\mathbf{z}_{k \mid k}$ and $\widehat{\mathbf{u}}_{k}$ are the approximately unbiased estimate of the actual $\mathbf{z}_{k}$ and $\mathbf{u}_{k}$, respectively, (16) can be rewritten as

$$
\begin{aligned}
\widetilde{\mathbf{z}}_{k \mid k-1} \equiv & \mathbf{z}_{k}-\mathbf{z}_{k \mid k-1} \approx \mathbf{z}_{k}^{\operatorname{lin}}-\mathbf{z}_{k \mid k-1}^{\operatorname{lin}} \\
= & \nabla_{\mathbf{z}} f_{k-1} \cdot\left(\mathbf{z}_{k-1}-\mathbf{z}_{k-1 \mid k-1}\right)+\nabla_{\mathbf{u}} f_{k-1} \\
& \cdot\left(\mathbf{u}_{k-1}-\widehat{\mathbf{u}}_{k-1}\right)+\mathbf{w}_{k-1} \\
= & \nabla_{\mathbf{z}} f_{k-1} \cdot \widetilde{\mathbf{z}}_{k-1 \mid k-1}+\nabla_{\mathbf{u}} f_{k-1} \cdot \widetilde{\mathbf{u}}_{k-1}+\mathbf{w}_{k-1} \\
= & \widetilde{\mathbf{z}}_{k \mid k-1}^{\operatorname{lin}},
\end{aligned}
$$

where $\widetilde{\mathbf{z}}_{k \mid k} \equiv \mathbf{z}_{k}-\mathbf{z}_{k \mid k}$ and $\widetilde{\mathbf{u}}_{k} \equiv \mathbf{u}_{k}-\widehat{\mathbf{u}}_{k}$. Consequently, the covariance matrix of $\mathbf{z}_{k \mid k-1}$ is given by

$$
\begin{aligned}
& \mathbf{P}_{k \mid k-1}^{z} \equiv E\left(\widetilde{\mathbf{z}}_{k \mid k-1} \widetilde{\mathbf{z}}_{k \mid k-1}^{T}\right) \\
& \approx\left[\begin{array}{ll}
\nabla_{\mathbf{z}} f_{k-1} & \nabla_{\mathbf{u}} f_{k-1}
\end{array}\right]\left[\begin{array}{cc}
\mathbf{P}_{k-1 \mid k-1}^{z} & \mathbf{P}_{k-1}^{z u} \\
\mathbf{P}_{k-1}^{u z} & \mathbf{P}_{k-1}^{u}
\end{array}\right]\left[\begin{array}{c}
\left(\nabla_{\mathbf{z}} f_{k-1}\right)^{T} \\
\left(\nabla_{\mathbf{u}} f_{k-1}\right)^{T}
\end{array}\right] \\
& \quad+\mathbf{G}_{k-1} \equiv \mathbf{P}_{k \mid k-1}^{z, \operatorname{lin}},
\end{aligned}
$$

where $\mathbf{P}_{k \mid k}^{z} \equiv E\left(\widetilde{\mathbf{z}}_{k \mid k} \widetilde{\mathbf{z}}_{k \mid k}^{T}\right), \mathbf{P}_{k}^{u} \equiv E\left(\widetilde{\mathbf{u}}_{k} \widetilde{\mathbf{u}}_{k}^{T}\right), \mathbf{P}_{k}^{u z}=\left(\mathbf{P}_{k}^{z u}\right)^{T} \equiv$ $E\left(\widetilde{\mathbf{u}}_{k} \widetilde{\mathbf{z}}_{k \mid k}^{T}\right)$.

For the steps of input identification and measurement update in Algorithm 1 (the standard GDF algorithm), these matrices, $\widetilde{\mathbf{R}}_{k}, \mathbf{J}_{k}, \widehat{\mathbf{u}}_{k}, \mathbf{P}_{k}^{u}, \mathbf{K}_{k}, \mathbf{z}_{k \mid k}, \mathbf{P}_{k \mid k}^{z}$, and $\mathbf{P}_{k}^{z u}$, can still be used as the linear approximations just as the method in [12]. The extended GDF algorithm is shown in Algorithm 2. 


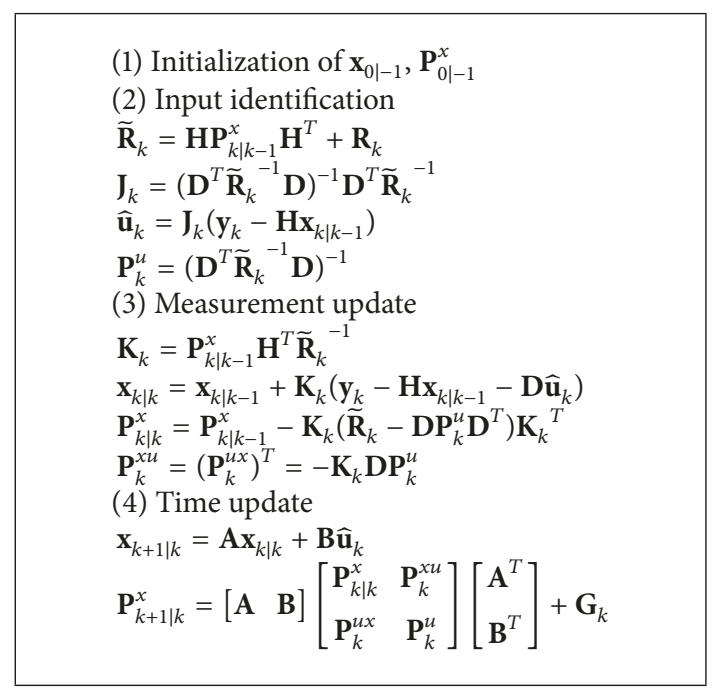

Algorithm 1: The algorithm of GDF. Note. $\widehat{\mathbf{u}}_{k}$ is the estimate of input $\mathbf{u}_{k} ; \mathbf{P}_{k \mid k}^{x}=E\left[\widetilde{\mathbf{x}}_{k \mid k} \widetilde{\mathbf{x}}_{k \mid k}^{T}\right], \mathbf{P}_{k}^{u}=E\left[\widetilde{\mathbf{u}}_{k} \widetilde{\mathbf{u}}_{k}^{T}\right],\left(\mathbf{P}_{k}^{x u}\right)^{T}=\mathbf{P}_{k}^{u x}=E\left[\widetilde{\mathbf{u}}_{k} \widetilde{\mathbf{x}}_{k \mid k}^{T}\right]$, and $\mathbf{P}_{k \mid k-1}^{x}=E\left[\widetilde{\mathbf{x}}_{k \mid k-1} \widetilde{\mathbf{x}}_{k \mid k-1}^{T}\right]$ with $\widetilde{\mathbf{x}}_{k \mid k}=\mathbf{x}_{k}-\mathbf{x}_{k \mid k}$ and $\widetilde{\mathbf{u}}_{k}=\mathbf{u}_{k}-\widehat{\mathbf{u}}_{k}$.

If the three important sensitivity matrices $\nabla_{\mathbf{z}} f_{k}, \nabla_{\mathbf{u}} f_{k}$, and $\nabla_{\mathbf{z}} h_{k}$ are indeed linear, EGDF is identical to GDF. These sensitivity matrices are referred to as [32].

It is obvious that the idea of the EGDF algorithm is excited by the idea of the EKF algorithm. The nonlinear state transmission equation (11) and measurement equation (12) are linearized with the first-order Taylor expansion to make the standard GDF algorithm suitable for identifying the extended state and unknown input of the approximate linear dynamic system. Moreover, the EGDF algorithm has a more strict mathematics derivation and observes the definitions of prior PDF (probability density function) and posterior PDF in Bayesian framework compared with the algorithm proposed by Lei et al. [26]. In addition, the EGDF algorithm has two advantages compared with another popular algorithm called A-DEKF proposed by Naets et al. [28]. One is that the EGDF procedure does not need prior information on expected value and covariance of the force, while the force covariance plays an important role in the estimate of the unknown state and force in A-DEKF. And the other advantage is that the EGDF algorithm has less computational efforts and better computational stability due to the smaller size of the extended state vector including no any forces.

3.3. Data Fusion of Acceleration and Displacement in EGDF. In engineering applications, accelerometers are most often used in structural dynamics. However, it has been demonstrated that the GDF approach based on limited number of acceleration measurements is inherently unstable which leads to the so-called spurious low-frequency drift in the estimates of the force and the displacements [22]. The drift is caused by acceleration's insensitivity to any quasi-static component in the input force $[21,22]$. Although some regularization methods or postsignal processing schemes can be adopted to deal with the drift in the identified results [33-36], they

$$
\begin{aligned}
& \text { (1) Initialization of } \mathbf{z}_{0 \mid-1}, \mathbf{P}_{0 \mid-1}^{z} \\
& \text { (2) Input identification } \\
& \widetilde{\mathbf{R}}_{k}=\nabla_{\mathbf{z}} h_{k} \cdot \mathbf{P}_{k \mid k-1}^{z} \cdot\left(\nabla_{\mathbf{z}} h_{k}\right)^{T}+\mathbf{R}_{k} \\
& \mathbf{J}_{k}=\left(\mathbf{D}_{k}^{T} \widetilde{\mathbf{R}}_{k}^{-1} \mathbf{D}_{k}\right)^{-1} \mathbf{D}_{k}{ }^{T} \widetilde{\mathbf{R}}_{k}^{-1} \\
& \widehat{\mathbf{u}}_{k}=\mathbf{J}_{k}\left(\mathbf{y}_{k}-h_{k}\left(\mathbf{z}_{k \mid k-1}\right)\right) \\
& \mathbf{P}_{k}^{u}=\left(\mathbf{D}_{k}^{T} \widetilde{\mathbf{R}}_{k}^{-1} \mathbf{D}_{k}\right)^{-1} \\
& \text { (3) Measurement update } \\
& \mathbf{K}_{k}=\mathbf{P}_{k \mid k-1}^{z}\left(\nabla_{\mathbf{z}} h_{k}\right)^{T} \widetilde{\mathbf{R}}_{k}^{-1} \\
& \mathbf{z}_{k \mid k}=\mathbf{z}_{k \mid k-1}+\mathbf{K}_{k}\left(\mathbf{y}_{k}-h_{k}\left(\mathbf{z}_{k \mid k-1}\right)-\mathbf{D}_{k} \widehat{\mathbf{u}}_{k}\right) \\
& \mathbf{P}_{k \mid k}^{z}=\mathbf{P}_{k \mid k-1}^{z}-\mathbf{K}_{k}\left(\widetilde{\mathbf{R}}_{k}-\mathbf{D}_{k} \mathbf{P}_{k}^{u} \mathbf{D}_{k}^{T}\right) \mathbf{K}_{k}^{T} \\
& \mathbf{P}_{k}^{z u}=\left(\mathbf{P}_{k}^{u z}\right)^{T}=-\mathbf{K}_{k} \mathbf{D}_{k} \mathbf{P}_{k}^{u} \\
& \text { (4) Time update } \\
& \mathbf{z}_{k+1 \mid k}=f_{k}\left(\mathbf{z}_{k \mid k}, \widehat{\mathbf{u}}_{k}\right) \\
& \mathbf{P}_{k+1 \mid k}^{z} \equiv E\left(\widetilde{\mathbf{z}}_{k+1 \mid k} \widetilde{\mathbf{z}}_{k+1 \mid k}^{T}\right)=\left[\begin{array}{ll}
\nabla_{\mathbf{z}} f_{k} & \nabla_{\mathbf{u}} f_{k}
\end{array}\right]\left[\begin{array}{cc}
\mathbf{P}_{k \mid k}^{z} & \mathbf{P}_{k}^{z u} \\
\mathbf{P}_{k}^{u z} & \mathbf{P}_{k}^{u}
\end{array}\right] \\
& \cdot\left[\begin{array}{c}
\left(\nabla_{\mathbf{z}} f_{k}\right)^{T} \\
\left(\nabla_{\mathbf{u}} f_{k}\right)^{T}
\end{array}\right]+\mathbf{G}_{k}
\end{aligned}
$$

Algorithm 2: The algorithm of EGDF.

are not suitable for the real-time identification of coupled state/input/parameter. In [38], a method dealing with joint state and parameter identification was presented. And to resolve the issues related to the spurious low-frequency components in the displacement estimates, a strategy was proposed by including artificial displacement measurements into the observation vector. In this paper, the same strategy is adopted for coupled state/input/parameter identification. Partial measured displacements are added to the acceleration measurements since displacement and acceleration measurements contain low and high frequencies vibration characteristics, respectively. Therefore, the measured date $\mathbf{y}$ in the measurement equation (4) contains both partial structural displacement and acceleration measurements. The output influence matrix $\mathbf{H}$ and direct transmission matrix $\mathbf{D}$ in (5) are modified as

$$
\begin{aligned}
& \mathbf{H}=\left[\begin{array}{cc}
\mathbf{H}_{d} & \mathbf{0} \\
-\mathbf{H}_{a} \mathbf{M}^{-1} \mathbf{K} & -\mathbf{H}_{a} \mathbf{M}^{-1} \mathbf{C}
\end{array}\right], \\
& \mathbf{D}=\left[\begin{array}{c}
\mathbf{0} \\
\mathbf{H}_{a} \mathbf{M}^{-1} \mathbf{B}_{u}
\end{array}\right],
\end{aligned}
$$

where $\mathbf{H}_{d}$ represents the selection matrix of displacement measurements.

In addition, from the recursive estimation of unknown force in Algorithm 2, the matrix $\mathbf{D}_{k}$ should exist for the inverse computation of the unknown force with the weighted least-square estimation. This requires that the acceleration signals at unknown input locations must be measured.

\section{Numerical Studies}

In this section, two numerical examples are considered to evaluate the performance of the modified EGDF method. The 


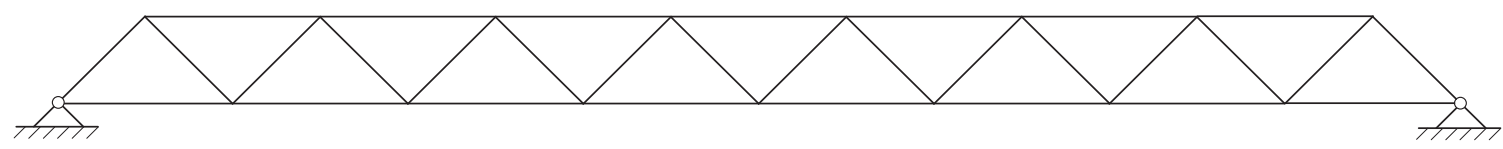

(a)

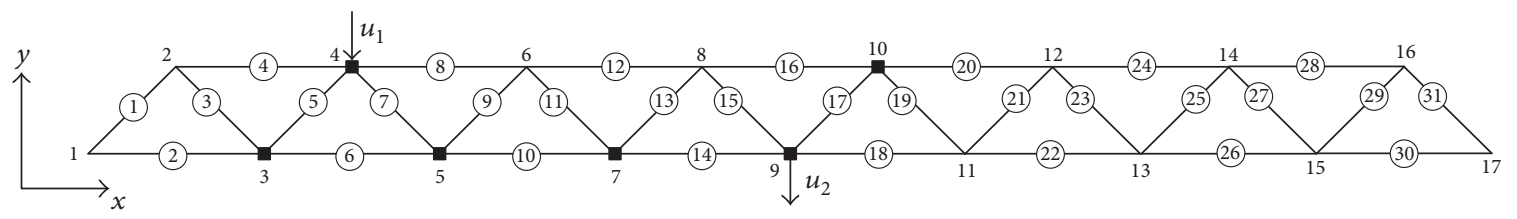

(b)

Figure 1: (a) Truss structure. (b) Finite element model of the truss and the sensor placement.

first example deals with a plane truss, and the other one is to identify the stiffness and damping of the vibration isolator for a single-stage isolation system.

4.1. Example 1: Plane Truss. A plane truss structure supported at two ends, as shown in Figure 1, is taken as the first numerical model. This truss structure is modeled using 31 planar truss finite elements with two DOFs, that is, lateral and vertical DOF at each free node. The cross-sectional area of all elements is $8.95 \times 10^{-5} \mathrm{~m}^{2}$, and all horizontal and vertical members are, respectively, of $2 \mathrm{~m}$ and $\sqrt{2} \mathrm{~m}$ length. Rayleigh damping is assumed, and the two damping coefficients are $\alpha=0.1523$ and $\beta=4.6503 \times 10^{-4}$. The mass density and elastic modulus of material are $7.85 \times 10^{3} \mathrm{kgm}^{-3}$ and $2 \times 10^{7} \mathrm{~Pa}$, respectively. The first eight natural frequencies of the structure are $0.15,0.41,0.86,1.02,1.39,1.77,2.14$, and $2.29 \mathrm{~Hz}$, respectively. Two external forces are applied at Node 4 and Node 9 . The force $u_{1}$ is a sinusoidal excitation as

$$
u_{1}=40 \sin (24 \pi t)+40 \sin (48 \pi t) .
$$

And the other force $u_{2}$ is a random input.

Assuming that the stiffness of truss elements 5, 7, 10, 14, 15, and 17 is an unknown parameter to be determined, the initial values are $759.5,633.0,1342.5,1163.5,759.5$, and $633.0 \mathrm{~N} / \mathrm{m}$, respectively. Thus, the extended state vector is $\left\{p_{1}, p_{2}\right.$, $\left.\ldots, p_{30}, \dot{p}_{1}, \dot{p}_{2}, \ldots, \dot{p}_{30}, k_{5}, k_{7}, k_{10}, k_{14}, k_{15}, k_{17}\right\}^{T}$. First, seven acceleration signals are measured for identification, which are the vertical acceleration signals at Nodes 3, 4, 5, 7, 9, and 10 and the horizontal acceleration signal at Node 9. Also $4 \%$ background environment noise is added to the measured signals. The initial extended state covariance $\mathbf{P}_{0 \mid-1}^{z}$ consisted of the covariance of the displacement, velocity vector $\left(\mathbf{P}_{0 \mid-1}^{d}\right.$, $\left.\mathbf{P}_{0 \mid-1}^{v}\right)$, and unknown stiffness vector $\left(\mathbf{P}_{0 \mid-1}^{\alpha}\right)$ and is assumed as

$$
\begin{aligned}
& \mathbf{P}_{0 \mid-1}^{d}=\operatorname{diag}\left\{10^{-16}, 10^{-16}, \ldots\right\}_{1 \times n \text { dof }}, \\
& \mathbf{P}_{0 \mid-1}^{v}=\operatorname{diag}\left\{10^{-8}, 10^{-8}, \ldots\right\}_{1 \times n \text { dof }}, \\
& \mathbf{P}_{0 \mid-1}^{\alpha}=\operatorname{diag}\left\{10^{14} \times 0.5^{2}, 10^{14} \times 0.5^{2}, \ldots\right\}_{1 \times 6},
\end{aligned}
$$

where $n$ dof $=30$ is the number of the truss structure. The covariance matrices of the measurement noise vector $\mathbf{v}_{k}$ and

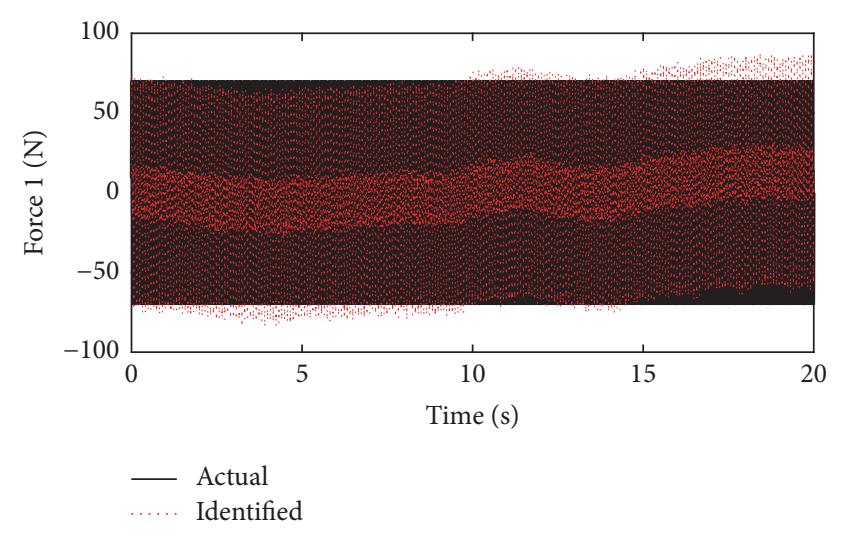

FIGURE 2: The actual and identified results of force $u_{1}$.

the system noise vector $\mathbf{w}_{k}$ are chosen to be $\mathbf{R}_{k}=0.1 \mathbf{I}_{6}$ and $\mathbf{G}_{k}=\operatorname{diag}\left\{[0,0, \ldots]_{1 \times 2 \times n \text { dof }},\left[10^{8}, 10^{8}, \ldots\right]_{1 \times 6}\right\}$, respectively. Based on the EGDF algorithm, the two forces are identified, and the identified curves are, respectively, plotted in Figures 2 and 3 with comparison to the actual curves of forces. From the curves, it is found that both of the two identified force values suffer from the low frequency drift compared to the actual values. All the structural displacement and velocity are identified. The identified results of the vertical states at Node 7 and Node 11 are, respectively, shown in Figures 4-5 together with the theoretical results. It is found that both of the identified velocities are accurate while the identified displacements cause the low-frequency drifts.

To solve the above drift problem, partial measured displacements are added to the measured response. The vertical displacements of Node 3 and 10 are measured together with the above seven acceleration. Figures 6-9 show the comparisons of the identified values of force, displacement, and velocity with their exact values, respectively. It is found that the so-called drift can be avoided by the modified EGDF with data fusion. The identified curves of structural responses and forces are all in good agreement with the curves of their exact values.

Figure 10 shows the convergences of the identified stiffness for three cases of different $\mathbf{G}_{k}$ values, that is, 


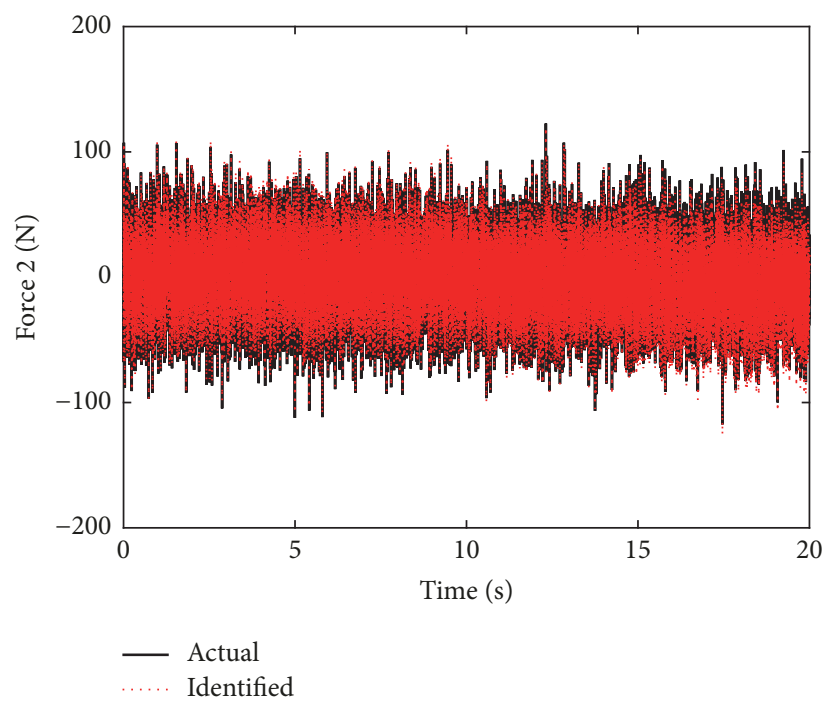

(a)

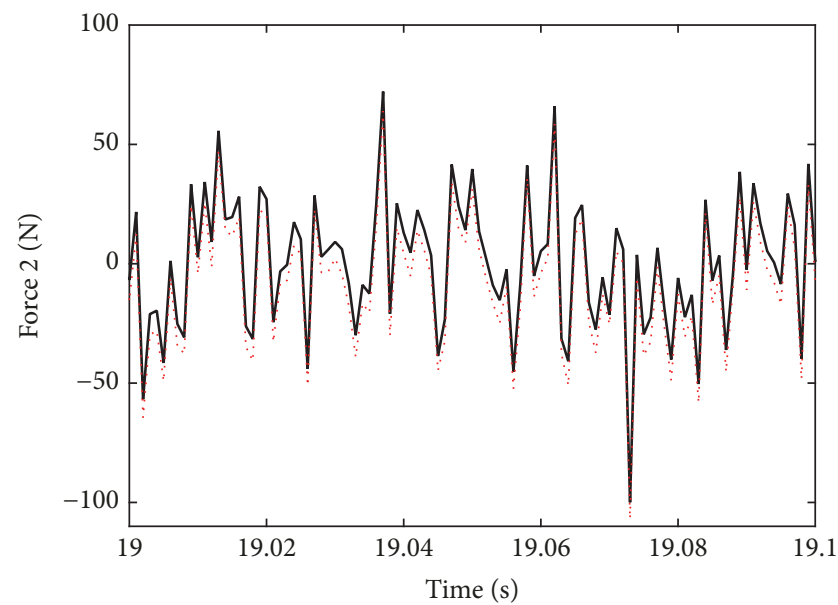

(b)

FIgURE 3: (a) The actual and identified results of force $u_{2}$. (b) Zoom-in view of a segment (1-1.1s) of (a).

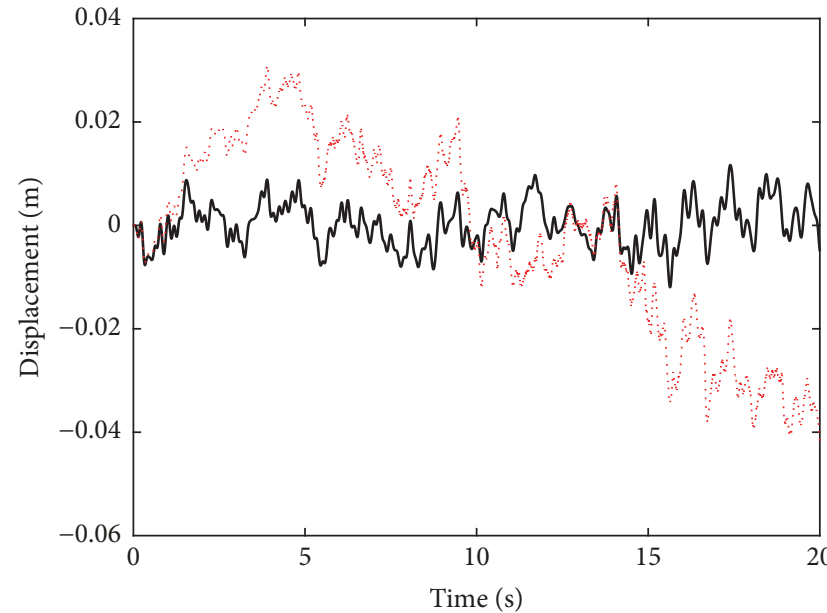

(a)

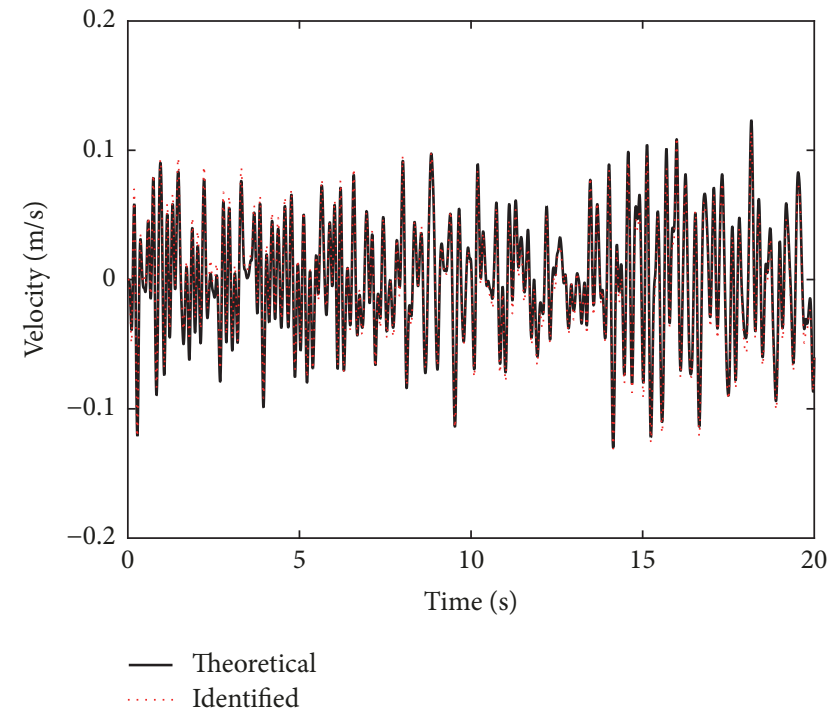

(b)

Figure 4: (a) The theoretical and identified vertical displacement at Node 7. (b) The theoretical and identified vertical velocity at Node 7.

$\mathbf{G}_{k}=\operatorname{diag}\left\{[0,0, \ldots]_{1 \times 2 \times n \operatorname{dof}},\left[10^{6}, 10^{6}, \ldots\right]_{1 \times 6}\right\}, \operatorname{diag}\{[0,0$, $\left.\ldots]_{1 \times 2 \times n \text { dof }},\left[10^{8}, 10^{8}, \ldots\right]_{1 \times 6}\right\}$, and $\operatorname{diag}\left\{[0,0, \ldots]_{1 \times 2 \times n \text { dof }}\right.$, $\left.\left[10^{10}, 10^{10}, \ldots\right]_{1 \times 6}\right\}$. Firstly, it is observed that all the stiffness values are updated quickly to their actual values and then walk randomly around the corresponding actual values. Secondly, it can be seen that the identified stiffness values are more accurate with less oscillation when the $\mathbf{G}_{k}$ value is smaller. The identified stiffness values are effective for the two cases of smaller $\mathbf{G}_{k}$ values, and it is comprehensible that a small model error can bring an accurate identified value with a little oscillation. Finally, the identified stiffness values are obtained by computing the means from $10 \mathrm{~s}$ to $20 \mathrm{~s}$ in Figure 10 for $\mathbf{G}_{k}=\operatorname{diag}\left\{[0,0, \ldots]_{1 \times 2 \times n \text { dof }},\left[10^{8}, 10^{8}, \ldots\right]_{1 \times 6}\right\}$ case, which are listed in Table 1 . By the above identified results including unknown forces, structural states, and unknown stiffness parameters, it can be concluded that the modified EGDF method with data fusion is suitable for the coupled state/input/parameter identification for linear structural systems.

4.2. Example 2: Single-Stage Isolation System. The singlestage isolation system is shown as Figure 11. The foundation is the steel plate, whose mass density, elastic modulus of material, and Poisson's ratio are $7.8 \times 10^{3} \mathrm{kgm}^{-3}, 2 \times 10^{11} \mathrm{~Pa}$, and 0.3 , respectively. And the size of the plate is 1200-600$20 \mathrm{~mm}$ (length-width-thickness). The two sides of the plate 


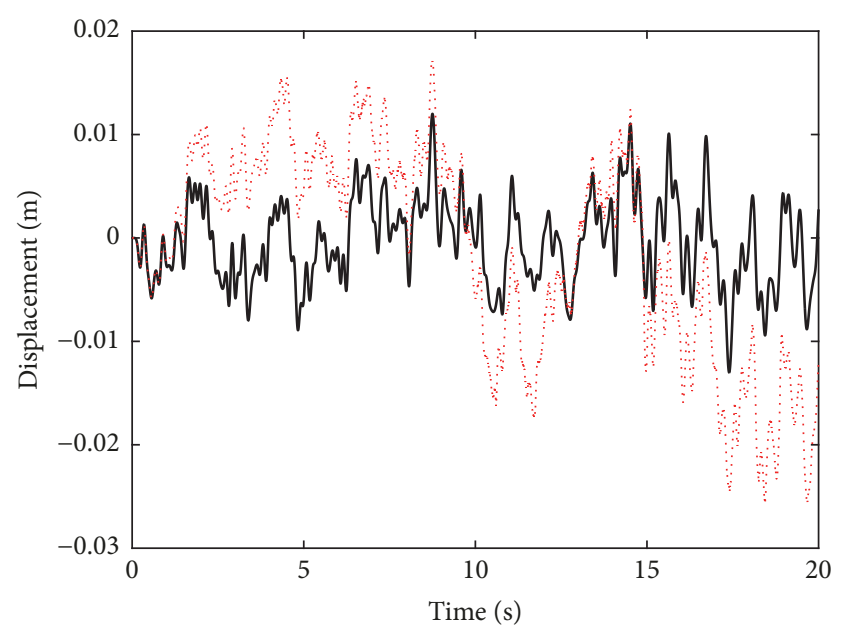

(a)

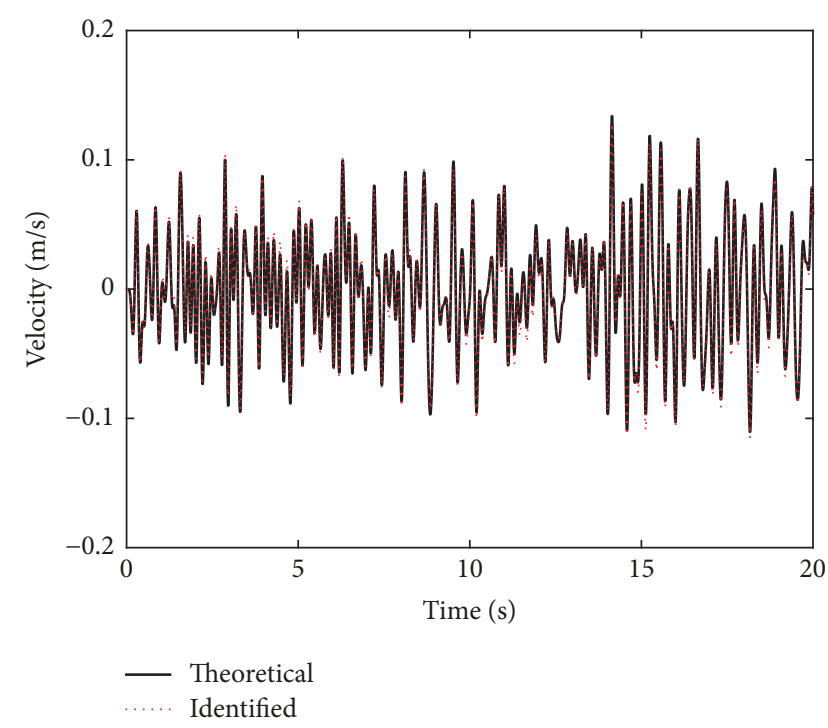

(b)

FIgURE 5: (a) The theoretical and identified vertical displacement at Node 11. (b) The theoretical and identified vertical velocity at Node 11.

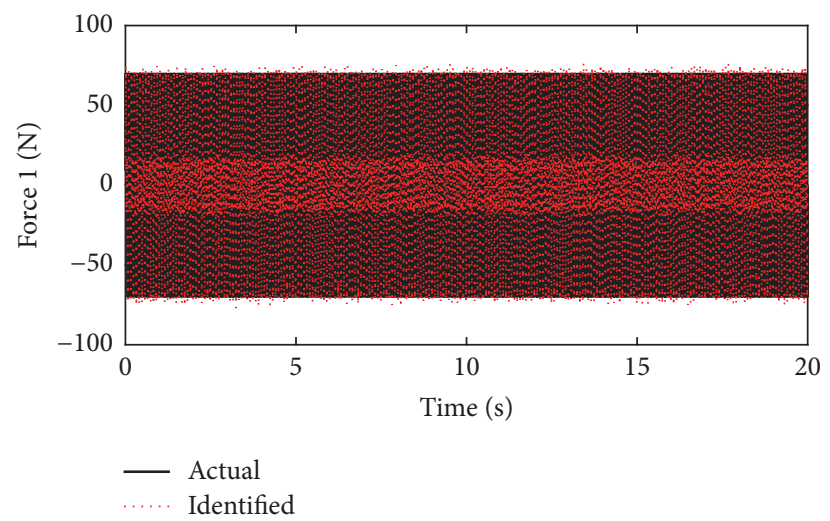

FIGURE 6: The actual and identified results of force $u_{1}$ by data fusion.

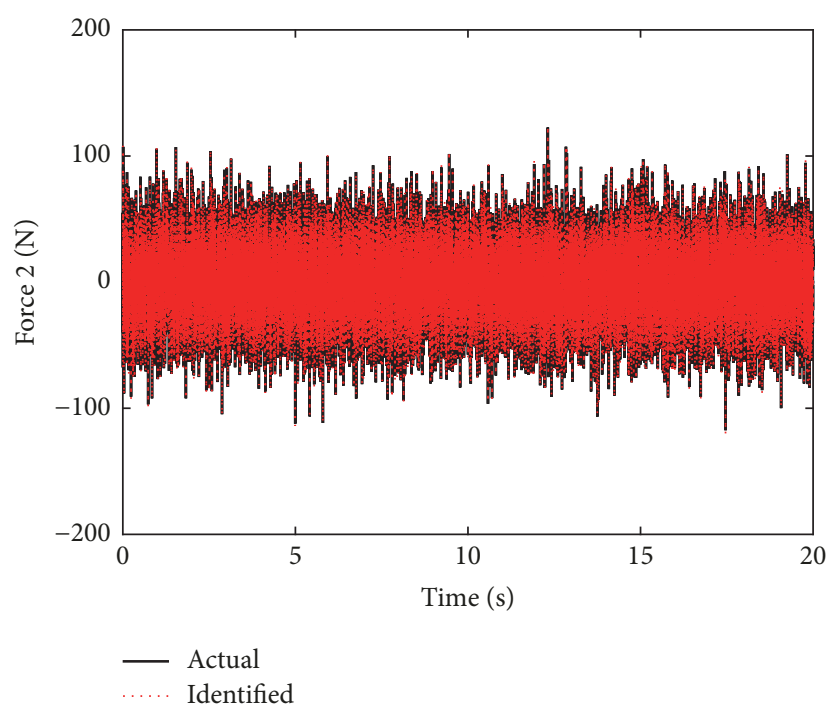

(a)

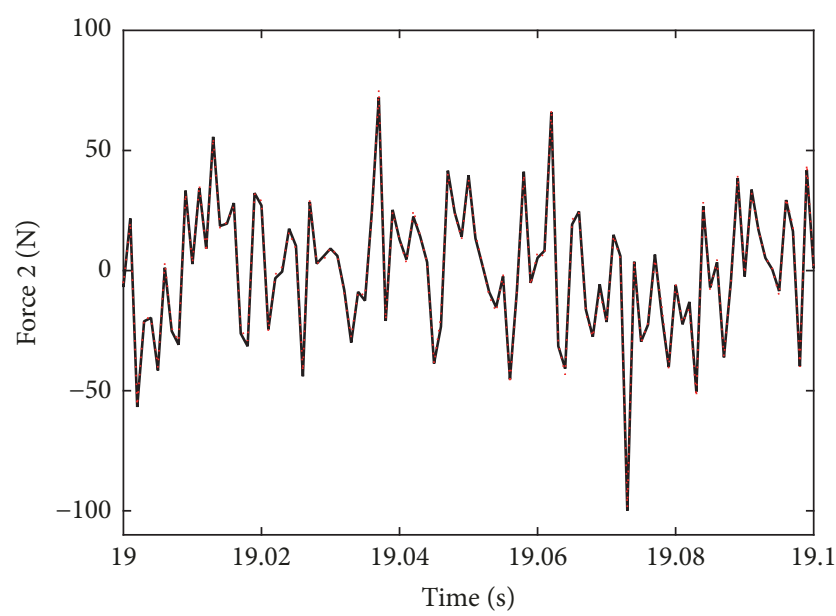

(b)

Figure 7: (a) The actual and identified results of force $u_{2}$ by data fusion. (b) Zoom-in view of a segment (19-19.1 s) of (a). 


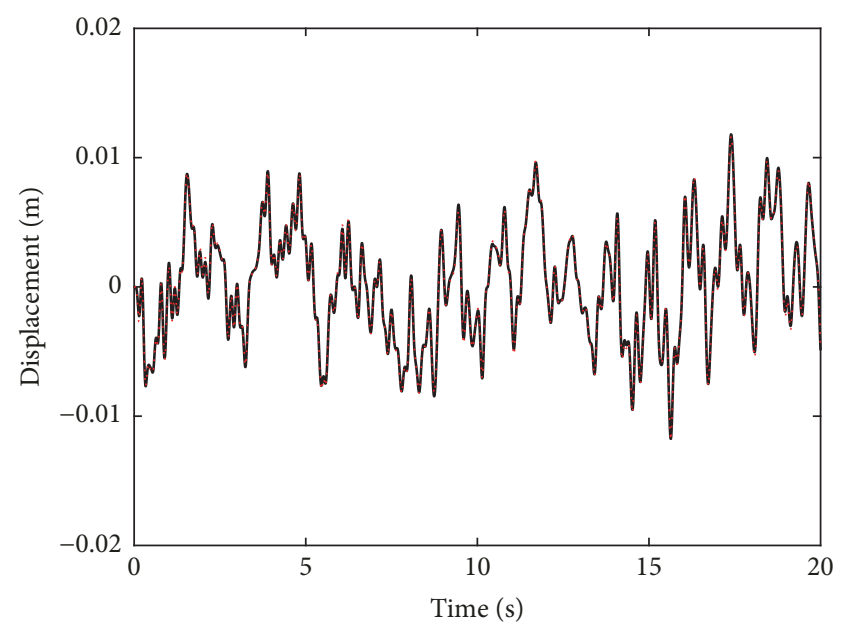

(a)

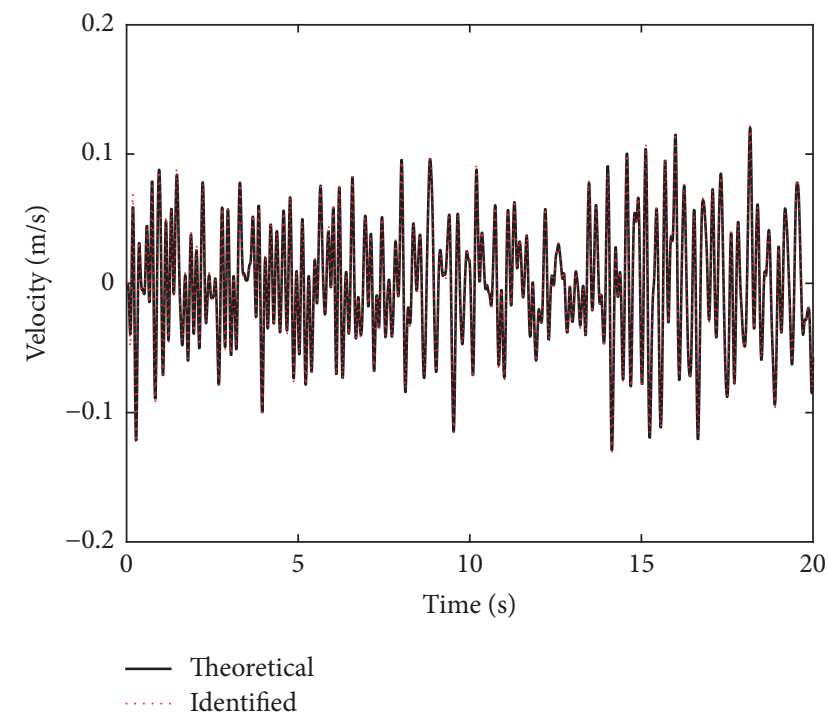

(b)

FIGURE 8: (a) The theoretical and identified vertical displacement at Node 7 by data fusion. (b) The theoretical and identified vertical velocity at Node 7.

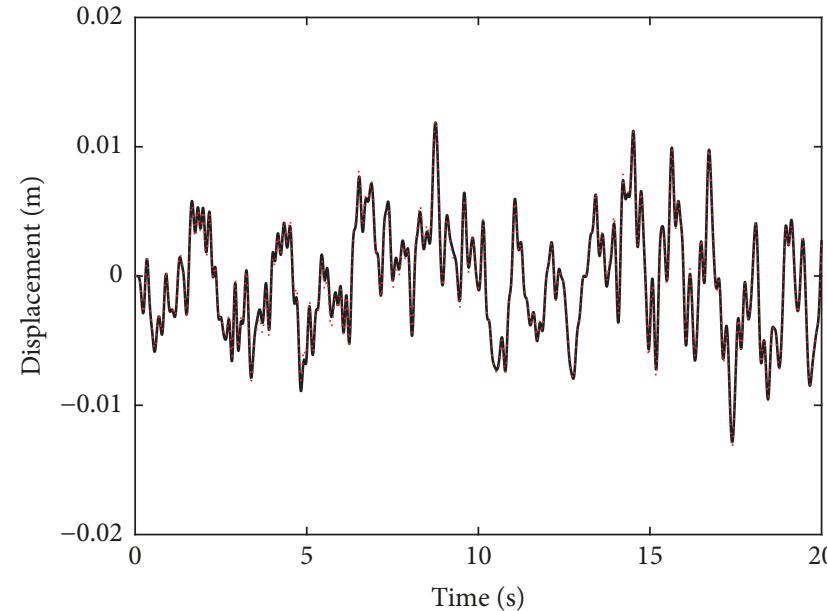

(a)

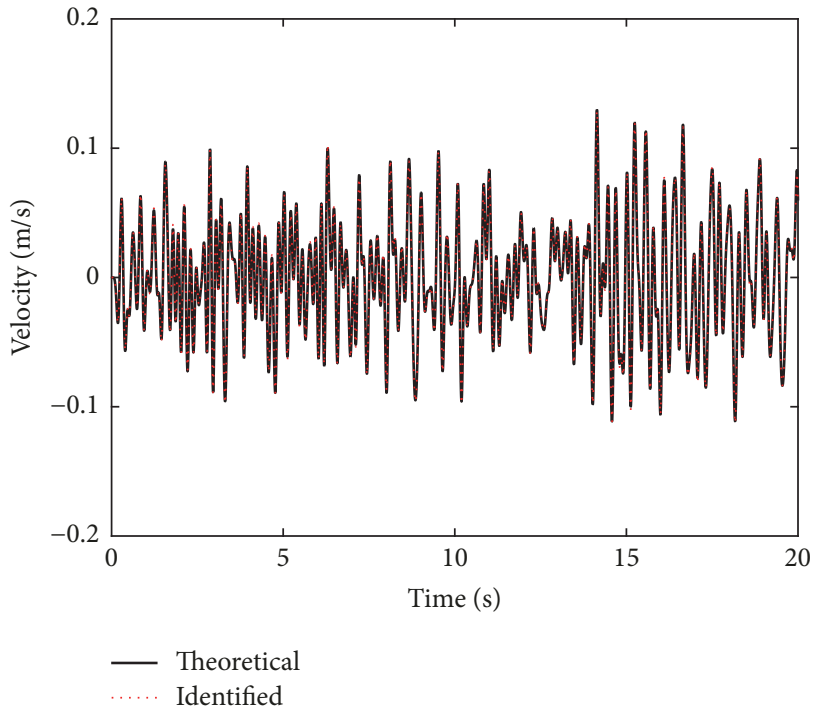

(b)

Figure 9: (a) The theoretical and identified vertical displacement at Node 11 by data fusion. (b) The theoretical and identified vertical velocity at Node 11.

TABLE 1: Comparisons of identified element stiffness parameters of the truss.

\begin{tabular}{|c|c|c|c|c|c|c|}
\hline Element number & 5 & 7 & 10 & 14 & 15 & 17 \\
\hline $\mathrm{Ki}(\mathrm{N} / \mathrm{m})$ exact & 1265.9 & 1265.9 & 895 & 895 & 1265.9 & 1265.9 \\
\hline $\begin{array}{l}\mathrm{ki}(\mathrm{N} / \mathrm{m}) \\
\text { identified }\end{array}$ & 1263.2 & 1268.0 & 894.407 & 898.436 & 1267.5 & 1265.8 \\
\hline Error (\%) & -0.21 & 0.17 & -0.07 & 0.38 & 0.13 & -0.01 \\
\hline
\end{tabular}




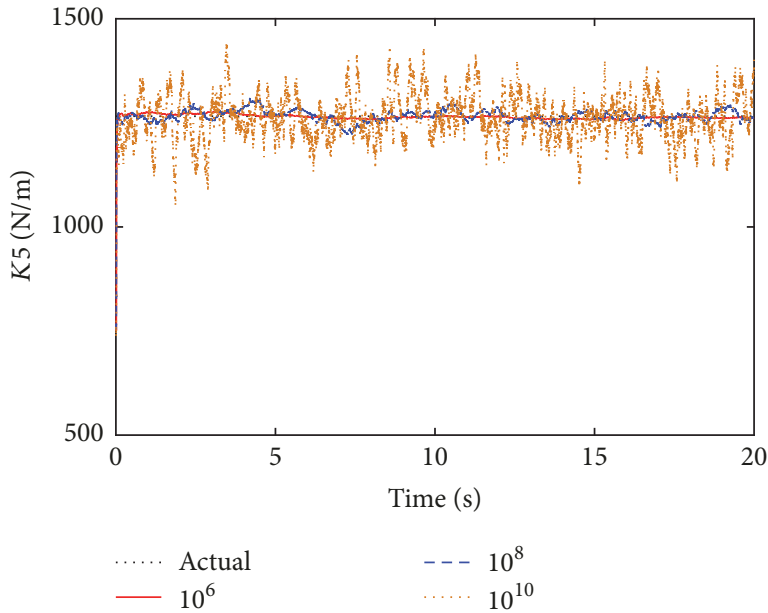

(a)

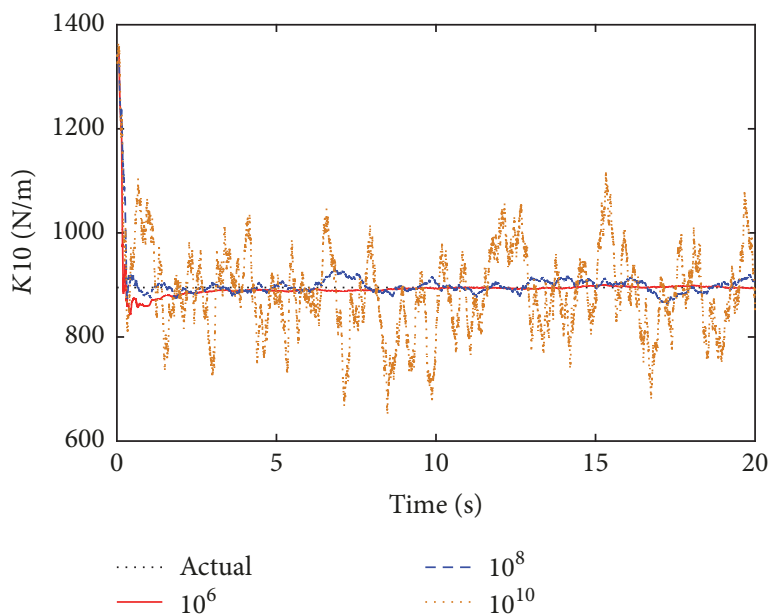

(c)

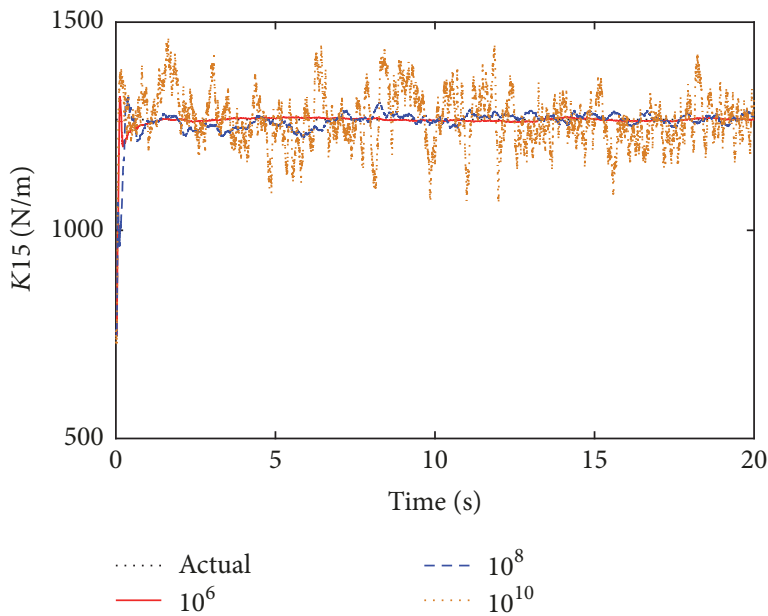

(e)

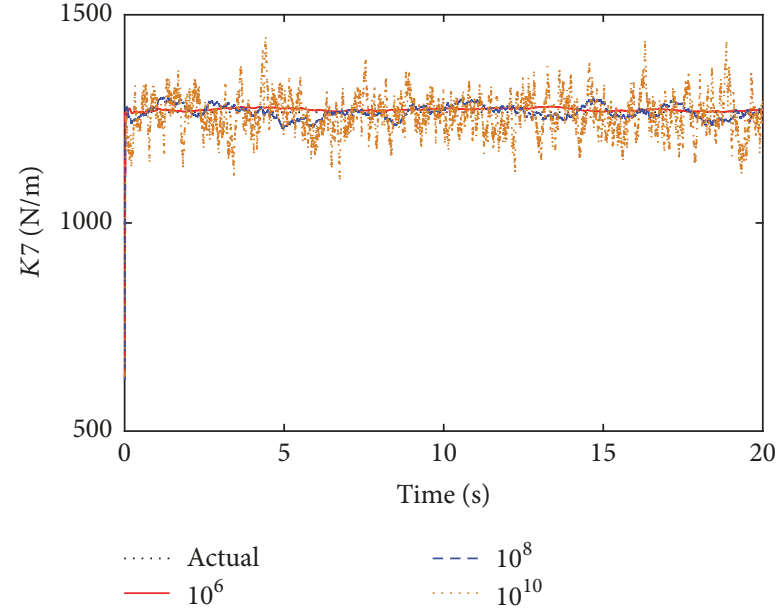

(b)

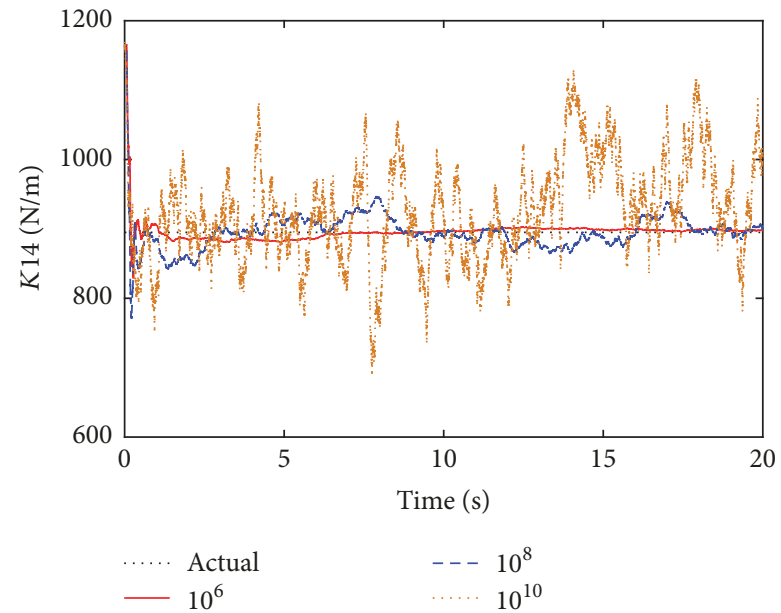

(d)

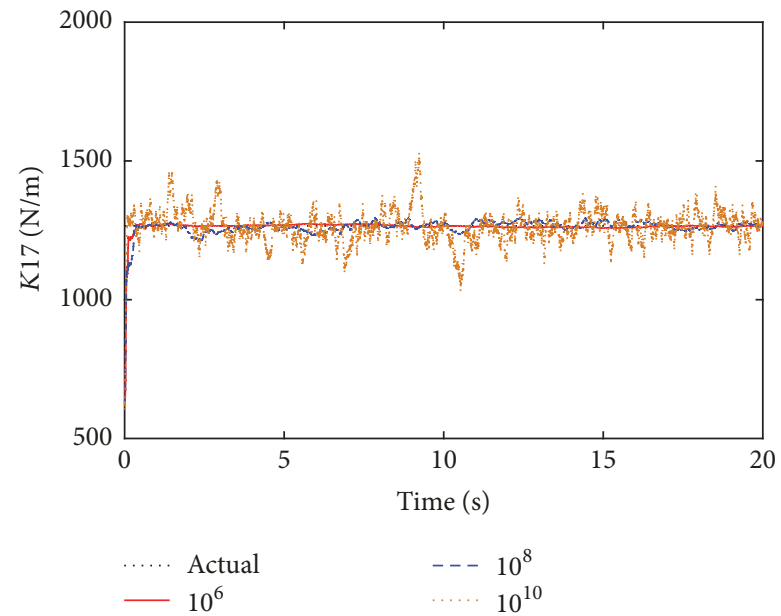

(f)

Figure 10: The actual and identified stiffness for Elements 5, 7, 10, 14, 15, and 17. 


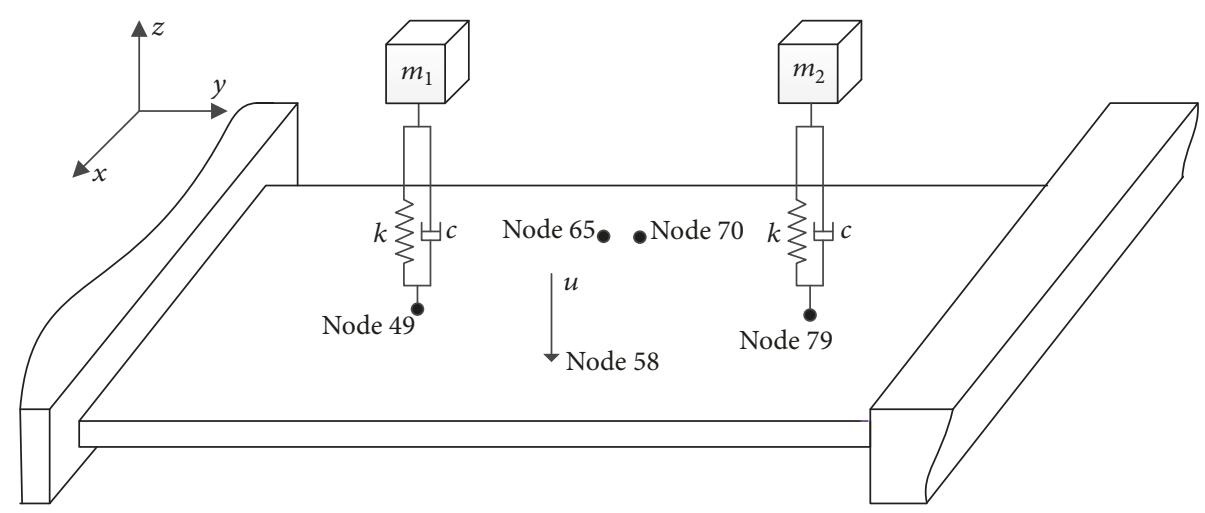

FIGURE 11: The single-stage isolation system.

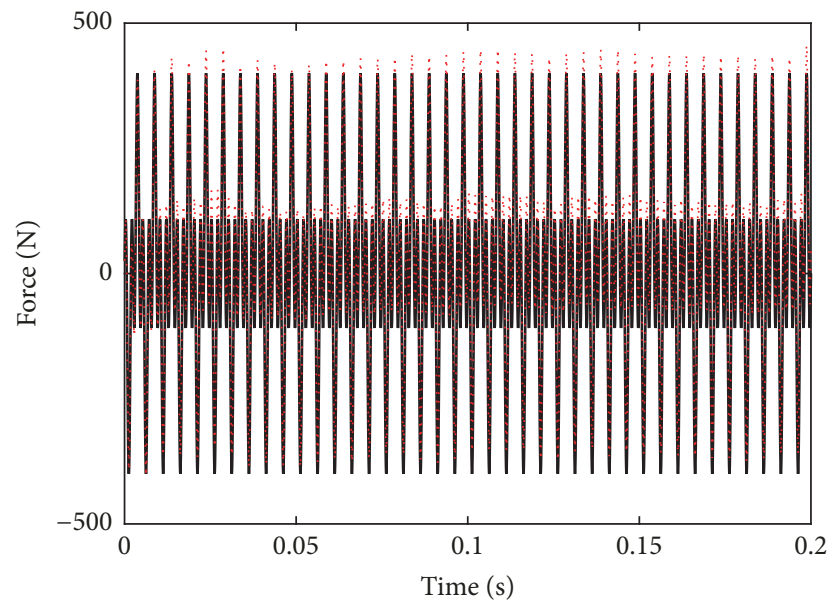

(a)

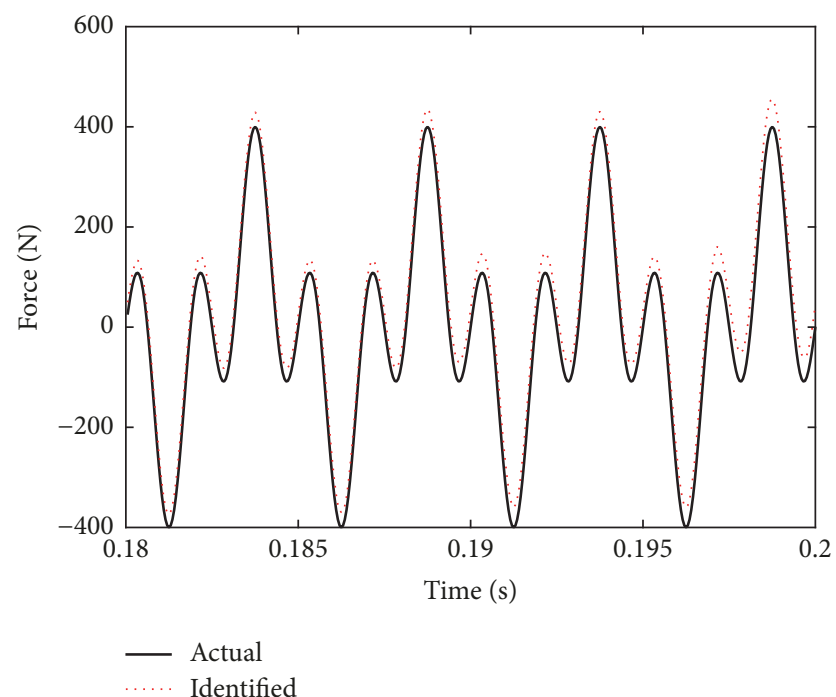

(b)

FIgURE 12: The actual and identified results of force $u$.

are fixed boundaries. Two same vibration isolators are used to mount two machines on the steel plate, and the stiffness and damping values are $k=10^{7} \mathrm{~N} / \mathrm{m}$ and $c=100 \mathrm{Ns} / \mathrm{m}$, respectively. The two machines have the mass values $m_{1}=$ $200 \mathrm{~kg}$ and $m_{2}=250 \mathrm{~kg}$. The steel plane is divided into $12 \times 6$ (length $\times$ width) plane elements for FE analysis. One external force is applied at Node 58 and it has the following form as

$$
u(t)=400 \sin (400 \pi t) \cos (800 \pi t) .
$$

Assuming that the stiffness and damping values of vibration isolators are unknown parameters to be determined, their initial values are $2 \times 10^{7} \mathrm{~N} / \mathrm{m}$ and $300 \mathrm{Ns} / \mathrm{m}$, respectively. Four acceleration signals of $z$-axis direction at Nodes 49,58 , 65 , and 79 are assumed to be measured for identification. Also $4 \%$ of background environment noise is added to the measured signals. The initial extended state covariance $\mathbf{P}_{0 \mid-1}^{z}$ consisted of the covariance of the displacement, velocity vector $\left(\mathbf{P}_{0 \mid-1}^{d}, \mathbf{P}_{0 \mid-1}^{v}\right)$, and unknown stiffness vector $\left(\mathbf{P}_{0 \mid-1}^{\alpha}\right)$ and is assumed as

$$
\begin{aligned}
& \mathbf{P}_{0 \mid-1}^{d}=\operatorname{diag}\left\{10^{-14}, 10^{-14}, \ldots\right\}_{1 \times n \text { dof }}, \\
& \mathbf{P}_{0 \mid-1}^{v}=\operatorname{diag}\left\{10^{-6}, 10^{-6}, \ldots\right\}_{1 \times n \text { dof }}, \\
& \mathbf{P}_{0 \mid-1}^{\alpha}=\operatorname{diag}\left\{10^{20}, 10^{10}\right\},
\end{aligned}
$$

where $n$ dof $=387$ is the number of the truss structure. The covariance matrices of the measurement noise vector $\mathbf{v}_{k}$ and the system noise vector $\mathbf{w}_{k}$ are chosen to be $\mathbf{R}_{k}=$ $0.1 \mathbf{I}_{4}$ and $\mathbf{G}_{k}=\operatorname{diag}\left\{[0,0, \ldots]_{1 \times 2 \times n \text { dof }}, 10^{8}, 10^{-2}\right\}$, respectively. If only four acceleration responses are used for identification, the drift can be found in identified force and displacement which are shown in Figures 12-13. To circumvent the above drift problem, the displacement signals 


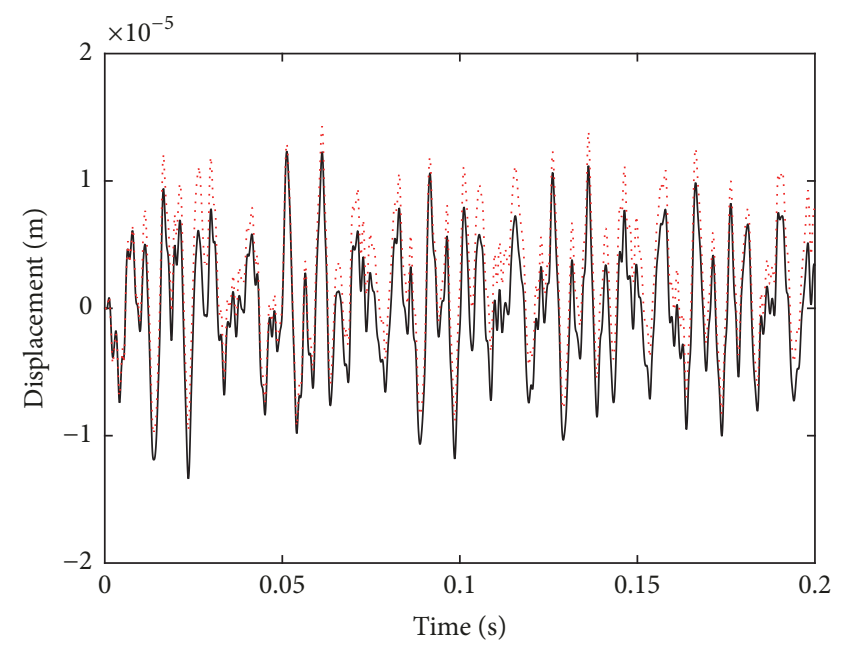

(a)

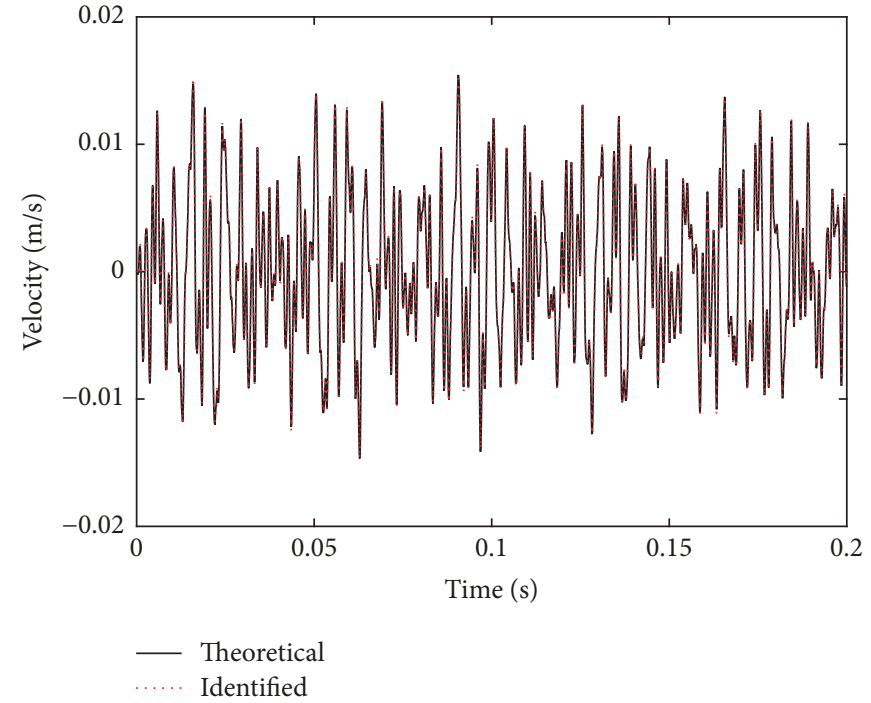

(b)

FIgURE 13: (a) The theoretical and identified vertical displacement at Node 70. (b) The theoretical and identified vertical velocity at Node 70.

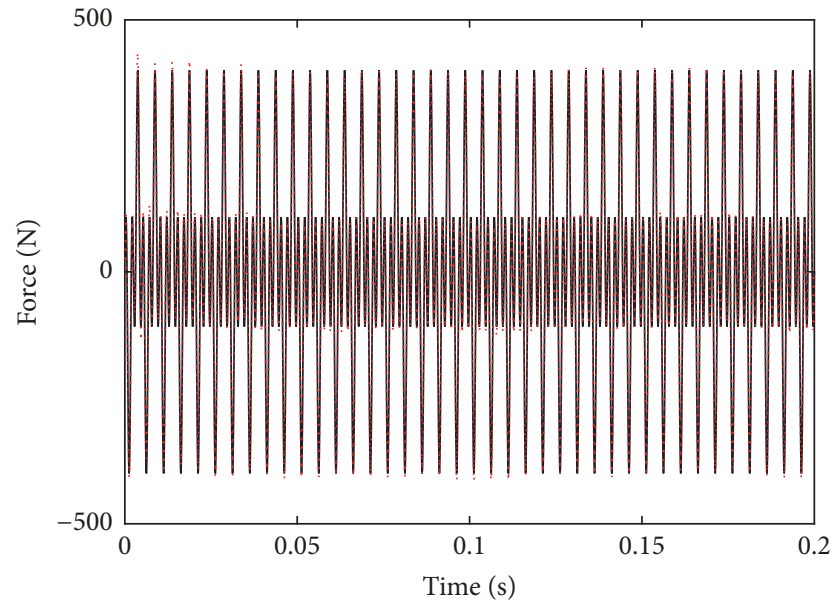

(a)

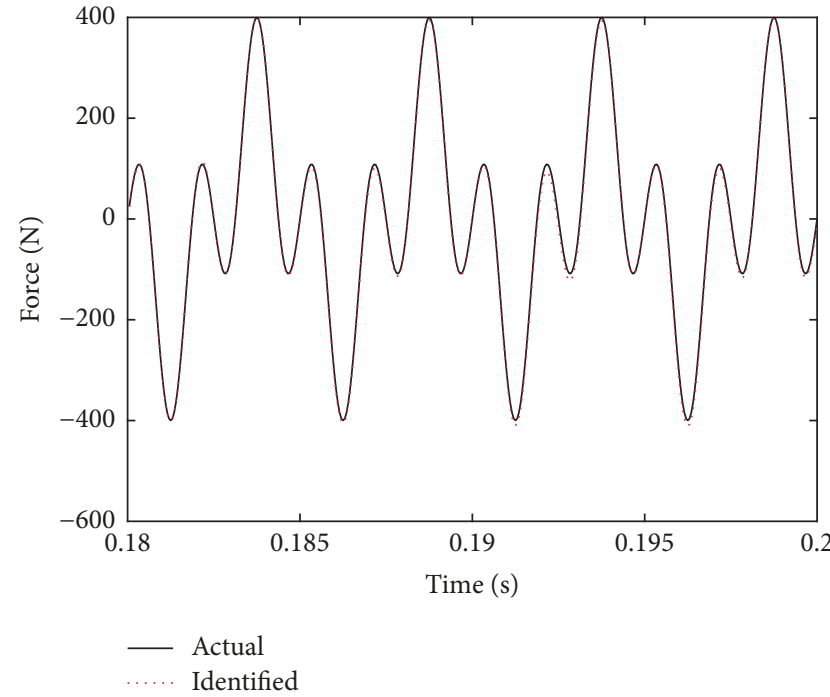

(b)

FIGURE 14: The actual and identified results of force $u$ by data fusion.

of $z$-axis direction at Node 63 are added to the above acceleration measurements for data fusion. The comparisons of the identified structural state and input with their exact values are shown in Figures 14-15. It can be seen that the previous low-frequency drifts in identified force and displacement disappeared by the modified EGDF method with data fusion. Moreover, in Figure 16, the unknown stiffness and damping values are identified for three cases of different $\mathbf{G}_{k}$ values, that is, $\mathbf{G}_{k}=\operatorname{diag}\left\{[0,0, \ldots]_{1 \times 2 \times n \text { dof }}, 10^{6}, 10^{-4}\right\}$, $\operatorname{diag}\left\{[0,0, \ldots]_{1 \times 2 \times n \text { dof }}, 10^{8}, 10^{-2}\right\}$, and $\operatorname{diag}\left\{[0,0, \ldots]_{1 \times 2 \times n \text { dof }}\right.$, $\left.10^{10}, 10^{0}\right\}$. It also can be seen that the identified parameters are more accurate with less oscillation when the $\mathbf{G}_{k}$ value is smaller, and the identified stiffness values are effective for the two cases of smaller $\mathbf{G}_{k}$ values. Table 2 shows the convergent parameters for the case of $\mathbf{G}_{k}=$ $\operatorname{diag}\left\{[0,0, \ldots]_{1 \times 2 \times n \text { dof }}, 10^{8}, 10^{-2}\right\}$. The small identified errors together with the above identified force and states represent the fact that the modified EGDF method with data fusion is suitable for the coupled state/input/parameter identification for linear structural systems.

\section{Conclusions}

The conventional EGDF algorithm of coupled state/input/ parameter identification is unstable for estimated inputs and displacements based solely on acceleration measurements. 


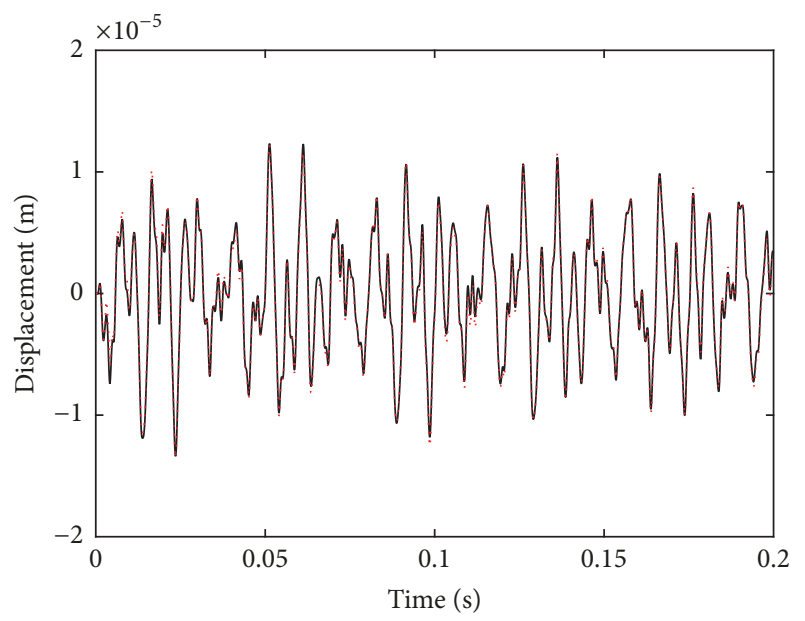

(a)

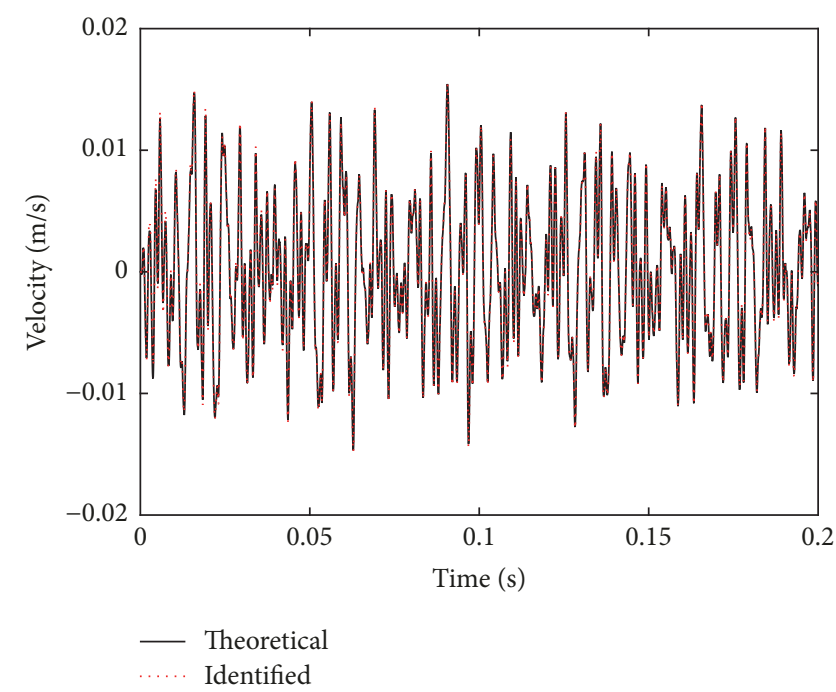

(b)

FIGURE 15: (a) The theoretical and identified vertical displacement at Node 70 by data fusion. (b) The theoretical and identified vertical velocity at Node 70.

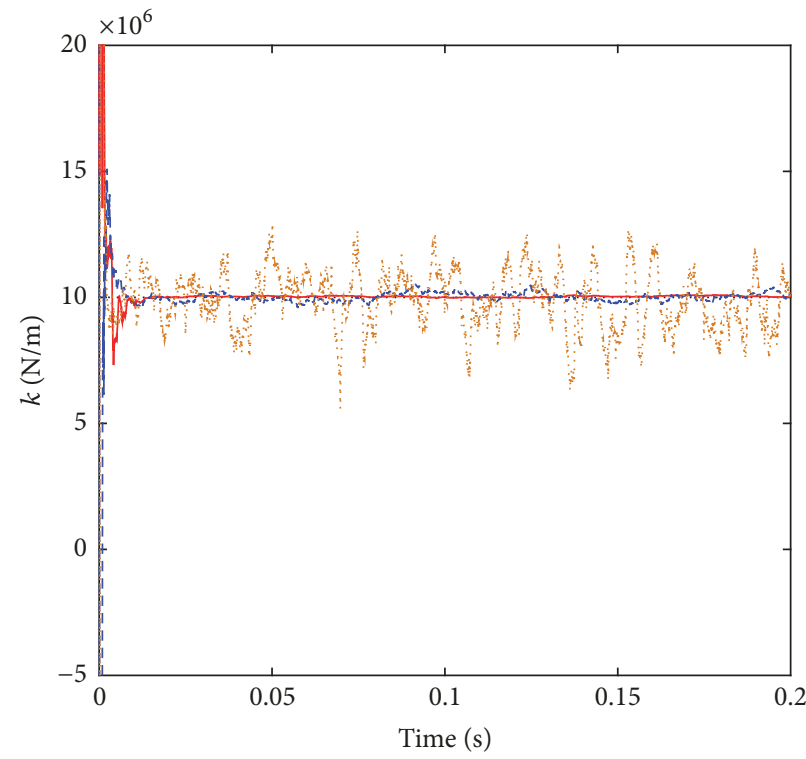

(a)

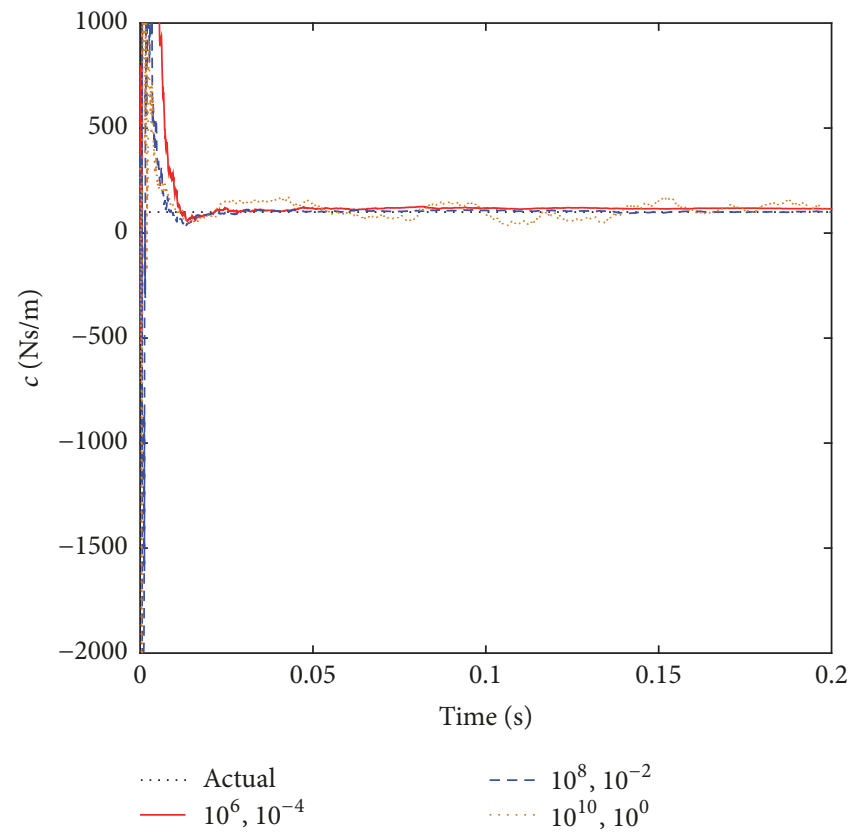

(b)

FIGURE 16: The actual and identified stiffness and damping of the vibration isolator.

TABLE 2: Comparisons of identified parameters.

\begin{tabular}{lcc}
\hline Identified parameter & $k(\mathrm{~N} / \mathrm{m})$ & $c(\mathrm{Ns} / \mathrm{m})$ \\
\hline Exact & $1 e 7$ & 100 \\
Identified & $1.0024 e 7$ & 98.6 \\
Error $(\%)$ & 0.24 & -1.4 \\
\hline
\end{tabular}

And the spurious low-frequency drift can be seen in the estimates of inputs and displacements, which is caused by acceleration's insensitivity to any quasi-static component in the input force. In this paper, a modified strategy of the EGDF algorithm based on data fusion of partially measured acceleration and displacement responses is adopted for linear structural systems, since displacement and acceleration measurements contain low and high frequencies vibration characteristics, respectively. Two numerical examples about a plane truss and a single-stage isolation system are conducted to verify the effectiveness of the proposed modified EGDF 
algorithm. The effect of the variances of the process noise is also tested for stability. Additionally, the EGDF method needs the acceleration response signals at unknown input locations to be measured for the inverse computation of the unknown force with the weighted least-square estimation.

\section{Conflicts of Interest}

The authors declare that they have no conflicts of interest.

\section{Acknowledgments}

This work was supported by the Technology Foundation of Nantong (GY12016043 and CP12015008).

\section{References}

[1] J. Ching, J. L. Beck, and K. A. Porter, "Bayesian state and parameter estimation of uncertain dynamical systems," Probabilistic Engineering Mechanics, vol. 21, no. 1, pp. 81-96, 2006.

[2] S. Gillijns and B. De Moor, "Unbiased minimum-variance input and state estimation for linear discrete-time systems," Automatica, vol. 43, no. 1, pp. 111-116, 2007.

[3] E. M. Hernandez, "A natural observer for optimal state estimation in second order linear structural systems," Mechanical Systems and Signal Processing, vol. 25, no. 8, pp. 2938-2947, 2011.

[4] M. Wu and A. W. Smyth, "Application of the unscented Kalman filter for real-time nonlinear structural system identification," Structural Control and Health Monitoring, vol. 14, no. 7, pp. 971990, 2007.

[5] D. Bernal, "Kalman filter damage detection in the presence of changing process and measurement noise," Mechanical Systems and Signal Processing, vol. 39, no. 1-2, pp. 361-371, 2013.

[6] J. Ching and J. L. Beck, "Real-time reliability estimation for serviceability limit states in structures with uncertain dynamic excitation and incomplete output data," Probabilistic Engineering Mechanics, vol. 22, no. 1, pp. 50-62, 2007.

[7] K. Erazo and E. M. Hernandez, "A model-based observer for state and stress estimation in structural and mechanical systems: experimental validation," Mechanical Systems and Signal Processing, vol. 43, no. 1-2, pp. 141-152, 2014.

[8] R. E. Kalman, "A new approach to linear filtering and prediction problems," Journal of Fluids Engineering, vol. 82, no. 1, pp. 35-45, 1960.

[9] A. H. Jazwinski, Stochastic Processes and Filtering Theory, Academic Press, New York, NY, USA, 1970.

[10] J. N. Yang, S. L. Lin, H. W. Huang, and L. Zhou, "An adaptive extended Kalman filter for structural damage identification," Structural Control and Health Monitoring, vol. 13, no. 4, pp. 849867, 2006.

[11] X. Liu, P. J. Escamilla-Ambrosio, and N. A. J. Lieven, "Extended Kalman filtering for the detection of damage in linear mechanical structures," Journal of Sound and Vibration, vol. 325, no. 4-5, pp. 1023-1046, 2009.

[12] J. Lin and Y. Zhang, "Nonlinear structural identification using extended Kalman filter," Computers \& Structures, vol. 52, no. 4, pp. 757-764, 1994.

[13] A. Corigliano and S. Mariani, "Parameter identification in explicit structural dynamics: Performance of the extended Kalman filter," Computer Methods Applied Mechanics and Engineering, vol. 193, no. 36-38, pp. 3807-3835, 2004.
[14] C. P. Fritzen and S. Zhu, "Updating of finite element models by means of measured information," Computers \& Structures, vol. 40, no. 2, pp. 475-486, 1991.

[15] S. Gillijns and B. De Moor, "Unbiased minimum-variance input and state estimation for linear discrete-time systems with direct feedthrough," Automatica, vol. 43, no. 5, pp. 934-937, 2007.

[16] E. Lourens, C. Papadimitriou, S. Gillijns, E. Reynders, G. De Roeck, and G. Lombaert, "Joint input-response estimation for structural systems based on reduced-order models and vibration data from a limited number of sensors," Mechanical Systems and Signal Processing, vol. 29, pp. 310-327, 2012.

[17] K. Maes, A. W. Smyth, G. De Roeck, and G. Lombaert, "Joint input-state estimation in structural dynamics," Mechanical Systems and Signal Processing, vol. 70-71, pp. 445-466, 2016.

[18] K. Maes, K. Van Nimmen, E. Lourens et al., "Validation of joint input-state estimation for force identification and response estimation in structural dynamics," in Proceedings of the 8th European Workshop on Structural Health Monitoring (EWSHM '16), Bilbao, Spain, July 2016.

[19] E. Lourens, E. Reynders, G. De Roeck, G. Degrande, and G. Lombaert, "An augmented Kalman filter for force identification in structural dynamics," Mechanical Systems and Signal Processing, vol. 27, no. 1, pp. 446-460, 2012.

[20] F. Naets, R. Pastorino, J. Cuadrado, and W. Desmet, "Online state and input force estimation for multibody models employing extended Kalman filtering," Multibody System Dynamics, vol. 32, no. 3, pp. 317-336, 2014.

[21] F. Naets, J. Cuadrado, and W. Desmet, "Stable force identification in structural dynamics using Kalman filtering and dummymeasurements," Mechanical Systems and Signal Processing, vol. 50-51, pp. 235-248, 2015.

[22] S. Eftekhar Azam, E. Chatzi, and C. Papadimitriou, "A dual Kalman filter approach for state estimation via output-only acceleration measurements," Mechanical Systems and Signal Processing, vol. 60, pp. 866-886, 2015.

[23] S. E. Azam, E. Chatzi, C. Papadimitriou, and A. Smyth, "Experimental validation of the Kalman-type filters for online and real-time state and input estimation," Journal of Vibration and Control, vol. 23, no. 15, pp. 2494-2519, 2015.

[24] I. Kolmanovsky, I. Sivergina, and J. Sun, "Simultaneous input and parameter estimation with input observers and set-membership parameter bounding: theory and an automotive application," International Journal of Adaptive Control and Signal Processing, vol. 20, no. 5, pp. 225-246, 2006.

[25] J. N. Yang, S. Pan, and H. Huang, "An adaptive extended Kalman filter for structural damage identifications II: Unknown inputs," Structural Control and Health Monitoring, vol. 14, no. 3, pp. 497521, 2007.

[26] Y. Lei, Y. Jiang, and Z. Xu, "Structural damage detection with limited input and output measurement signals," Mechanical Systems and Signal Processing, vol. 28, pp. 229-243, 2012.

[27] Y. Lei, Y. Wu, and T. Li, "Identification of non-linear structural parameters under limited input and output measurements," International Journal of Non-Linear Mechanics, vol. 47, no. 10, pp. 1141-1146, 2012.

[28] F. Naets, J. Croes, and W. Desmet, "An online coupled state/ input/parameter estimation approach for structural dynamics," Computer Methods Applied Mechanics and Engineering, vol. 283, pp. 1167-1188, 2015. 
[29] D. J. M. Fallais, S. Voormeeren, and E. Lourens, "Vibrationbased identification of hydrodynamic loads and system parameters for offshore wind turbine support structures," Energy Procedia, vol. 94, pp. 191-198, 2016.

[30] S. E. Azam, V. Dertimanis, E. Chatzi, and C. Papadimitriou, "Output-only schemes for joint input-state-parameter estimation of linear systems," in Proceedings of the 1st ECCOMAS Thematic Conference on Uncertainty Quantification in Computational Sciences and Engineering (UNCECOMP '15), pp. 497-510, Ceete Island, Greece, May 2015.

[31] V. Dertimanis, E. Chatzi, S. E. Azam, and C. Papadimitriou, "Output-only fatigue prediction of uncertain steel structures," in Proceedings of the 8th European Workshop on Structural Health Monitoring (EWSHM '16), Bilbao, Spain, July 2016.

[32] Z. Wan, T. Wang, Q. Huang, W. Zheng, and F. Gu, "Simultaneous excitation and parameter identification for non-linear structural systems," Journal of Vibroengineering, vol. 19, no. 6, pp. 3968-3980, 2017.

[33] B. Xu, J. He, R. Rovekamp, and S. J. Dyke, "Structural parameters and dynamic loading identification from incomplete measurements: Approach and validation," Mechanical Systems and Signal Processing, vol. 28, pp. 244-257, 2012.

[34] H. Zhu, L. Mao, and S. Weng, "Calculation of dynamic response sensitivity to substructural damage identification under moving load," Advances in Structural Engineering, vol. 16, no. 9, pp. 16211631, 2013.

[35] H.-P. Zhu, L. Mao, and S. Weng, "A sensitivity-based structural damage identification method with unknown input excitation using transmissibility concept," Journal of Sound and Vibration, vol. 333, no. 26, pp. 7135-7150, 2014.

[36] Q. Zhang, Ł. Jankowski, and Z. Duan, "Simultaneous identification of excitation time histories and parametrized structural damages," Mechanical Systems and Signal Processing, vol. 33, pp. 56-68, 2012.

[37] B. D. Anderson and J. B. Moore, Optimal Filtering, PrenticeHall, Englewood Cliffs, NJ, USA, 1979.

[38] E. N. Chatzi and C. Fuggini, "Online correction of drift in structural identification using artificial white noise observations and an unscented Kalman Filter," Smart Structures and Systems, vol. 16, no. 2, pp. 295-328, 2015. 


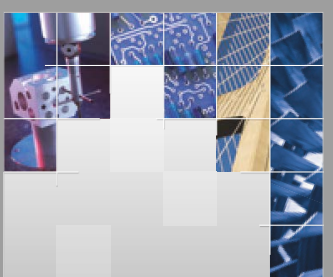

\section{Enfincering}
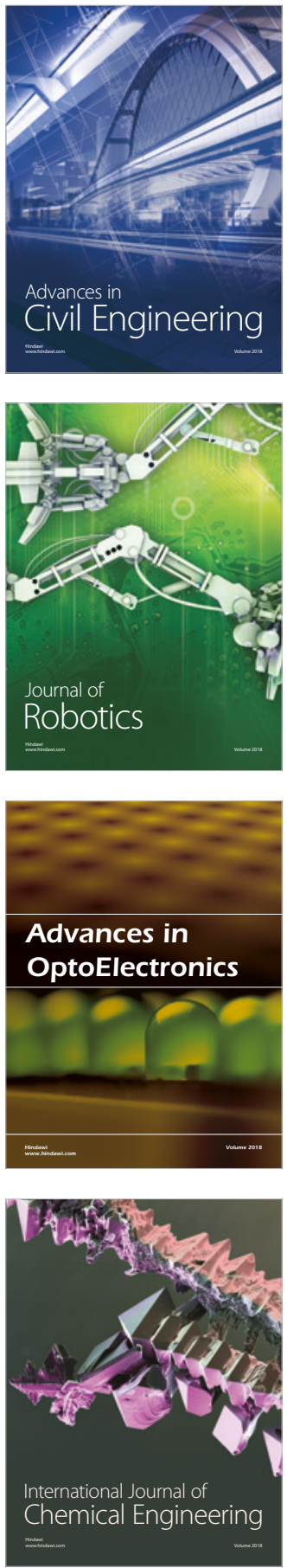

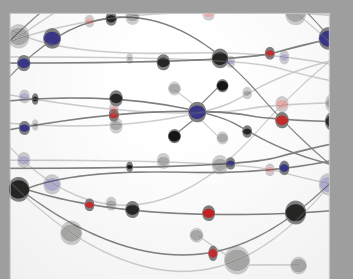

\section{Rotating \\ Machinery}

The Scientific World Journal

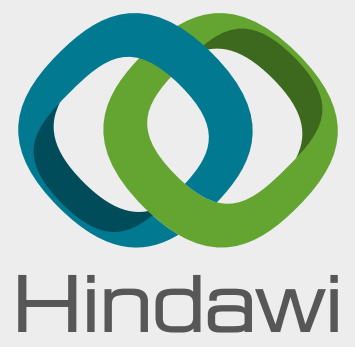

Submit your manuscripts at

www.hindawi.com
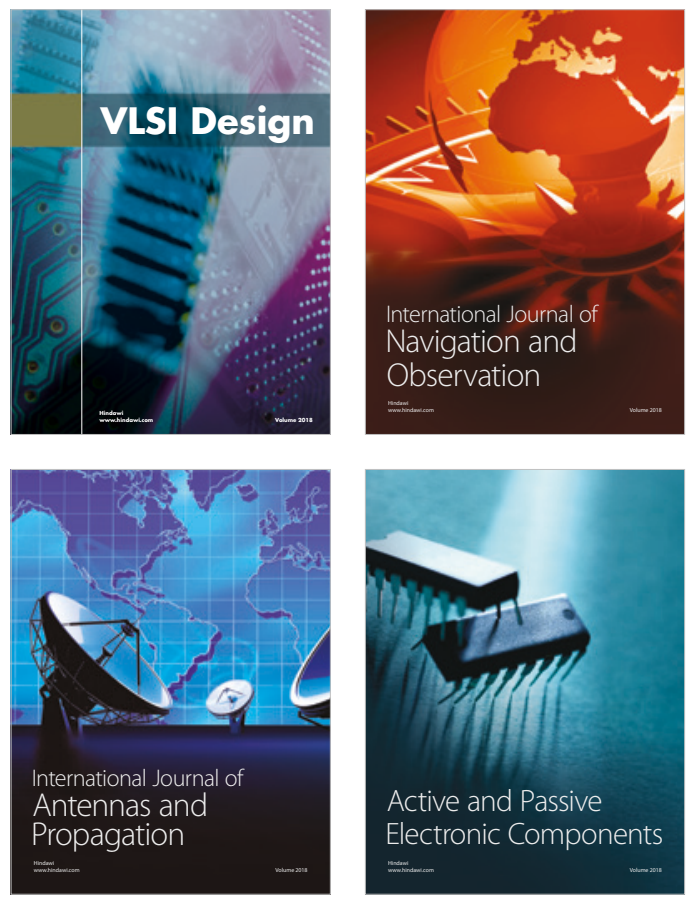
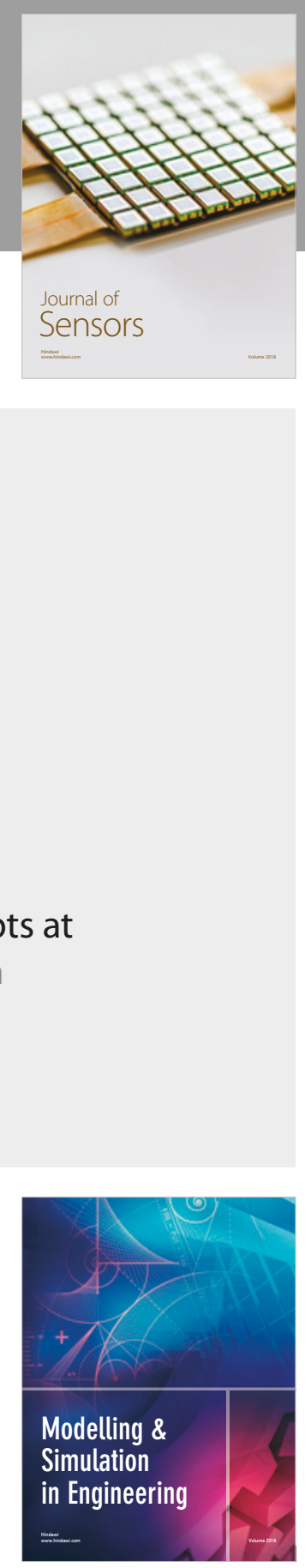

\section{Advances \\ Multimedia}
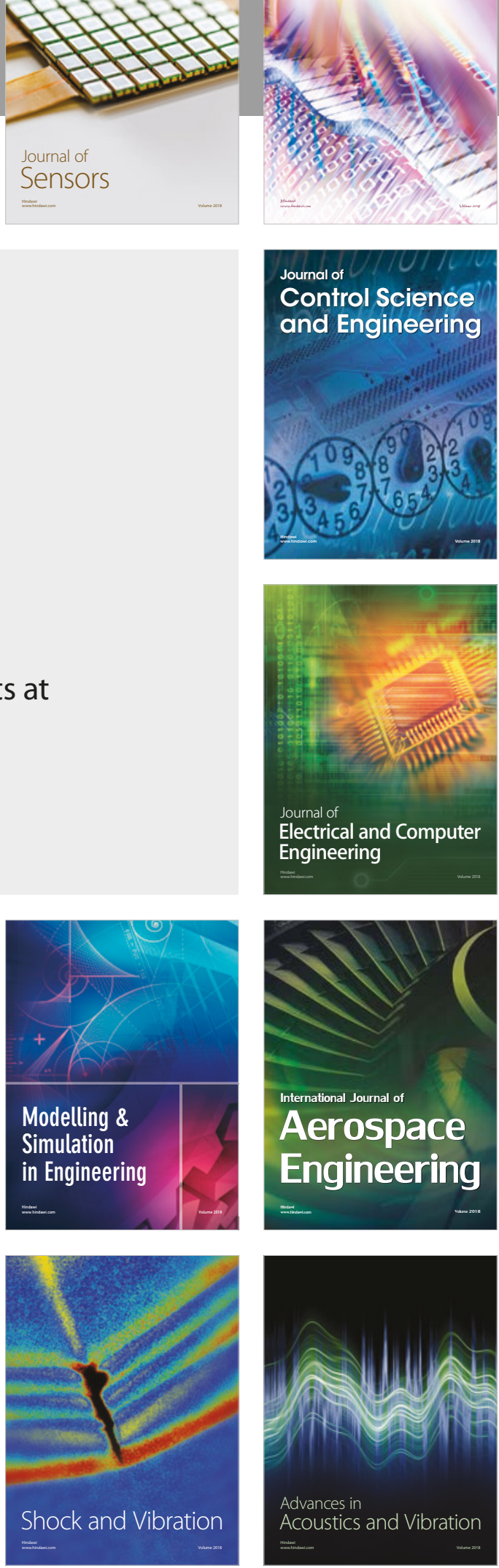